Brown Het-1309

\title{
Higher dimensional geometries related to Fuzzy odd-dimensional spheres
}

\author{
Sanjaye Ramgoolam \\ Department of Physics \\ Brown University \\ Providence, RI 02912 \\ ramgosk@het. brown.edu
}

We study $S O(m)$ covariant Matrix realizations of $\sum_{i=1}^{m} X_{i}^{2}=1$ for even $m$ as candidate fuzzy odd spheres following hep-th/0101001. As for the fuzzy four sphere, these Matrix algebras contain more degrees of freedom than the sphere itself and the full set of variables has a geometrical description in terms of a higher dimensional coset. The fuzzy $S^{2 k-1}$ is related to a higher dimensional coset $\frac{S O(2 k)}{U(1) \times U(k-1)}$. These cosets are bundles where base and fibre are hermitian symmetric spaces. The detailed form of the generators and relations for the Matrix algebras related to the fuzzy three-spheres suggests Matrix actions which admit the fuzzy spheres as solutions. These Matrix actions are compared with the BFSS, IKKT and BMN Matrix models as well as some others. The geometry and combinatorics of fuzzy odd spheres lead to some remarks on the transverse five-brane problem of Matrix theories and the exotic scaling of the entropy of 5-branes with the brane number.

June 2002 


\section{INTRODUCTION}

Fuzzy spheres provide many interesting solutions to Matrix Brane actions [1, 2, 3, 3, 4, 5] They have also been suggested to play a role in the context of a spacetime explanation of the stringy exclusion principle [6,7,8,9].

A class of odd-dimensional fuzzy spheres was defined in [10] and studied in more detail in [11]. The detailed $S O(m)$ decomposition of the Matrix algebras related to the fuzzy $S^{m}$ was given in [11]. For $m>2$ the Matrix algebras contain more representations than is necessary to describe functions on the sphere, and a projection is needed to get the desired traceless symmetric representations. The geometry of general even dimensional fuzzy spheres was studied in [12] and it was found that the Matrix algebras related to the fuzzy sphere of $2 k$ dimensions approaches, in a limit of large matrices, the algebra of functions on a higher dimensional space $S O(2 k+1) / U(k)$, the coset of $S O(2 k+1)$ by the right action of $U(k)$. This lead to the statement, for $k \geq 2$, that fluctuations around the fuzzy sphere solution can be described equivalently in two ways. On the one hand there is an abelian theory on the higher dimensional coset. On the other hand there is a non-abelian theory on the sphere. At finite $n$ this non-abelian theory on the fuzzy sphere has to be formulated on a commutative but non-associative algebra [11]. The structure constants of the associative algebra for the higher dimensional coset have been described more explicitly in [13]. The abelian theory on the higher dimensional coset, in the case $k=2$ has been further studied in [14]. A connection between the fuzzy four-sphere and the 4D quantum Hall effect [15] was found in [16] and higher even fuzzy spheres were used to explore $6 \mathrm{D}$ and $8 \mathrm{D}$ generalizations of the quantum Hall effect. The extra degrees of freedom of the fuzzy 4-sphere have been discussed in the context of uncertainty relations in [17].

In this paper we explore the extent to which the picture of physics on even dimensional fuzzy spheres developed in [12] can be extended to fuzzy odd spheres. We find that, as in the even case, there is an underlying higher dimensional geometry related to the Matrix algebra. For the case of the sphere $S^{2 k-1}$ with symmetry $S O(2 k)$, the relevant geometry is $\frac{S O(2 k)}{U(k-1) \times U(1)}$, the coset of $S O(2 k)$ by the right action of $U(k-1) \times U(1)$. We will discuss in detail the cases $k=2$ and $k=3$ in this paper but it appears that arguments leading to these cosets should generalize easily, especially the arguments in section 3.3 and 8.1.

The precise role of the $\frac{S O(2 k)}{U(k-1) \times U(1)}$ is, however, different from the role played by the $S O(2 k+1) / U(k)$ in the case of even dimensional spheres. To explain this, we need to 
recall some details about the construction of even and odd fuzzy spheres. In all cases one starts with matrices $X_{i}$, where $i$ transforms in the vector of $S O(m)$, and

$$
\sum_{i=1}^{m} X_{i}^{2}=1
$$

The $X_{i}$ are in $\operatorname{End}\left(\mathcal{R}_{n}\right)$, i.e Matrices which are transformations of the vector space $\mathcal{R}_{n}$, which is a representation of $S O(m)$. The $X_{i}$ in (1.1) are related by a rescaling to the $X_{i}$ used in the bulk of this paper. $\mathcal{R}_{n}$ is a vector space whose dimension $N$ depends on an integer $n$. The precise dependence of $N$ on $n$ can be found in [11]. The crucial difference between even and odd spheres is that, in the case of even spheres $\mathcal{R}_{n}$ is an irreducible representation of $S O(2 k+1)$, whereas in the case of odd spheres $\mathcal{R}_{n}$ is a direct sum of irreducible representations $\mathcal{R}_{n}^{+}$and $\mathcal{R}_{n}^{-}$of $S O(2 k)$, that is, $\mathcal{R}_{n}=\mathcal{R}_{n}^{+} \oplus \mathcal{R}_{n}^{-}$. The matrices $X_{i}$ are maps from $\mathcal{R}_{n}^{+}$to $\mathcal{R}_{n}^{-}$and vice versa from $\mathcal{R}_{n}^{-}$to $\mathcal{R}_{n}^{+}$.

For even fuzzy spheres, End $\left(\mathcal{R}_{n}\right)$ becomes commutative in the large $n$ limit and approaches the algebra of functions on a classical space $S O(2 k+1) / U(k)$. In the odd sphere case, both $\operatorname{End}\left(\mathcal{R}_{n}^{+}\right)$and $\operatorname{End}\left(\mathcal{R}_{n}^{-}\right)$become commutative in the large $n$ limit and approach the algebra of functions on $\frac{S O(2 k)}{U(k-1) \times U(1)}$. However the full Matrix algebra $\operatorname{End}\left(\mathcal{R}_{n}\right)$ remains non-commutative. In the case $k=2$, the relevant higher dimensional space $\frac{S O(4)}{U(1) \times U(1)}$ has a simpler description as $S^{2} \times S^{2}$ when we use the isomorphism $S O(4) \equiv S U(2) \times S U(2)$. Section 3 gives two ways to prove that $\operatorname{End}\left(\mathcal{R}_{n}^{ \pm}\right)$approaches the algebra of functions on $S^{2} \times S^{2}$ in the large $N$ limit. One uses the techniques of Kaluza-Klein reduction [18] or equivalently harmonic analysis on homogeneous spaces, as described for example in [19]. Another uses the analysis of the stabilizer group of a particular solution to the algebraic relations satisfied by the generators of the Matrix algebra. Section 8 proves, by similar methods, that the higher dimensional geometry related to the fuzzy five-sphere is $\frac{S O(6)}{U(2) \times U(1)}$.

In sections 4-7 we explore the fuzzy 3-sphere in more detail. Section 4 gives the relations between the generators of $\operatorname{End}\left(\mathcal{R}_{n}\right)$. Section 5 gives the large $n$ limit of these relations, showing, in particular, that while $\operatorname{End}\left(\mathcal{R}_{n}^{ \pm}\right)$become commutative, $\operatorname{End}\left(\mathcal{R}_{n}\right)$ remains non-commutative. Section 6 uses the relations derived in sections 4 and 5 to construct some Matrix actions which are solved by the fuzzy three-sphere Matrices. Section 7 discusses the physics of fluctuations around these solutions.

In section 9.1 we discuss the geometry of these cosets $\frac{S O(2 k)}{U(k-1) \times U(1)}$ further. In particular with describe some bundle structures they admit. For the even sphere case, the bundle structure of $S O(2 k+1) / U(k)$ was used to map abelian field theory on the fuzzy 
coset to non-abelian field theory on the fuzzy sphere base [12]. In the odd sphere case, the relevant higher dimensional geometry is not in general a bundle over the sphere, so the same strategy as [12] cannot be used to obtain a field theory on the sphere. However some bundle structures on the coset do exist and we describe them. An interesting feature is that base and fibre are both hermitian symmetric spaces, which come up in $N=2$ sigma models for example [20]. In section 9.3 we give a qualitative comparison of some basic counting of degrees of freedom which explains why it appears dificult to obtain an ordinary non-abelian theory on the fuzzy odd sphere. We observe some intriguing similarities to discussions of the entropy of M5-branes. Section 9 also describes some avenues for future research.

\section{Review of fuzzy odd spheres}

To get a fuzzy three sphere [10] we look for matrices which satisfy the equation $\sum_{i} X_{i}^{2}=c$, where $c$ is a constant. The matrices are constructed by starting with $V=V_{+} \oplus V_{-}$. The vector space $V_{+}$is the two-dimensional spinor representation of $S O(4)$ of positive chirality. The space $V_{-}$is the two-dimensional representation of negative chirality. The projector $P_{+}$acting on $V$ projects onto $V_{+}$, and $P_{-}$projects onto $V_{-}$. In terms of the $S O(4)=S U(2) \times S U(2)$ isomorphism, these have spins $\left(2 j_{L}, 2 j_{R}\right)=(1,0)$ and $\left(2 j_{L}, 2 j_{R}\right)=(0,1)$ respectively. For every odd integer $n$, the space $\operatorname{Sym}\left(V^{\otimes n}\right)$ ( which is the symmetrized $n$-fold tensor product space ) contains a subspace where there are $\frac{(n+1)}{2}$ factors of positive chirality and $\frac{(n-1)}{2}$ factors of negative chirality. This subspace is an irreducible representation of $S O(4)$ labelled by $\left(2 j_{L}, 2 j_{R}\right)=\left(\frac{(n+1)}{2}, \frac{(n-1)}{2}\right)$, which we will call $\mathcal{R}_{n}^{+}$. The projection operator in $\operatorname{End}\left(\operatorname{Sym}\left(V^{\otimes n}\right)\right)$ which projects onto this irrep is called $\mathcal{P}_{\mathcal{R}_{n}^{+}}$. In [10] it was called $\mathcal{P}_{\mathcal{R}^{+}}$, but here we are using notation which makes the $n$ dependence explicit. $\operatorname{Sym}\left(V^{\otimes n}\right)$ also contains a subspace where there are $\frac{(n+1)}{2}$ factors of negative chirality and $\frac{(n-1)}{2}$ factors of positive chirality. This subspace is an irreducible representation of $S O(4)$ labelled by $\left(2 j_{L}, 2 j_{R}\right)=\left(\frac{(n-1)}{2}, \frac{(n+1)}{2}\right)$, which we will call $\mathcal{R}_{n}^{-}$. The projector for this subspace is called $\mathcal{P}_{\mathcal{R}_{n}^{-}}$. The space $\mathcal{R}_{n}$ is defined to be a direct sum

$$
\mathcal{R}_{n}=\mathcal{R}_{n}^{+} \oplus \mathcal{R}_{n}^{-}
$$

The projector for this space is a sum

$$
\mathcal{P}_{\mathcal{R}_{n}}=\mathcal{P}_{\mathcal{R}_{n}^{+}}+\mathcal{P}_{\mathcal{R}_{n}^{-}}
$$


The Matrices $X_{i}$ are linear transformations of $\mathcal{R}_{n}$, i.e they are in $\operatorname{End}\left(\mathcal{R}_{n}\right)$.

$$
X_{i}=\mathcal{P}_{\mathcal{R}_{n}} \sum_{r} \rho_{r}\left(\Gamma_{i}\right) \mathcal{P}_{\mathcal{R}_{n}}
$$

More precisely, $X_{i}$ map $\mathcal{R}_{n}^{+}$to $\mathcal{R}_{n}^{-}$and $\mathcal{R}_{n}^{-}$to $\mathcal{R}_{n}^{+}$. This can be expressed by saying it is a sum of matrices in $\operatorname{Hom}\left(\mathcal{R}_{n}^{+}, \mathcal{R}_{n}^{-}\right)$and in $\operatorname{Hom}\left(\mathcal{R}_{n}^{+}, \mathcal{R}_{n}^{-}\right)$

$$
X_{i}=\mathcal{P}_{\mathcal{R}_{n}^{+}} X_{i} \mathcal{P}_{\mathcal{R}_{n}^{-}}+\mathcal{P}_{\mathcal{R}_{n}^{-}} X_{i} \mathcal{P}_{\mathcal{R}_{n}^{+}}
$$

In other words, if we arrange the vectors of $\mathcal{R}^{+}$along the upper rows of a column vector and those of $\mathcal{R}^{-}$along the lower rows, the matrices $X_{i}$ are non-zero in the off-diagonal blocks.

Let us prove that $\sum_{i} X_{i}^{2}$ commutes with generators of $S O(4)$.

$$
\begin{aligned}
& {\left[\sum_{i} X_{i}^{2}, \quad \mathcal{P}_{\mathcal{R}_{n}} \sum_{t} \rho_{t}\left(\Gamma_{k} \Gamma_{l}\right) \mathcal{P}_{\mathcal{R}_{n}}\right]} \\
& =\sum_{i}\left[X_{i}, \quad \mathcal{P}_{\mathcal{R}_{n}} \sum_{t} \rho_{t}\left(\Gamma_{k} \Gamma_{l}\right) \mathcal{P}_{\mathcal{R}_{n}}\right] X_{i} \\
& +X_{i}\left[X_{i}, \quad \mathcal{P}_{\mathcal{R}_{n}} \sum_{t} \rho_{t}\left(\Gamma_{k} \Gamma_{l}\right) \mathcal{P}_{\mathcal{R}_{n}}\right] \\
& =\sum_{i} \mathcal{P}_{\mathcal{R}_{n}} \sum_{r}\left[\rho_{r}\left(\Gamma_{i}\right), \quad \sum_{t} \rho_{t}\left(\Gamma_{k} \Gamma_{l}\right)\right] \mathcal{P}_{\mathcal{R}_{n}} \sum_{s} \rho_{s}\left(\Gamma_{i}\right) \mathcal{P}_{\mathcal{R}_{n}} \\
& +\mathcal{P}_{\mathcal{R}_{n}} \sum_{r} \rho_{r}\left(\Gamma_{i}\right) \mathcal{P}_{\mathcal{R}_{n}}\left[\sum_{s} \rho_{s}\left(\Gamma_{i}\right), \quad \sum_{t} \rho_{t}\left(\Gamma_{k} \Gamma_{l}\right)\right] \mathcal{P}_{\mathcal{R}_{n}} \\
& =\mathcal{P}_{\mathcal{R}_{n}} \sum_{r} \rho_{r}\left(\left[\Gamma_{i}, \quad \Gamma_{k} \Gamma_{l}\right]\right) \mathcal{P}_{\mathcal{R}_{n}} \sum_{s} \rho_{s}\left(\Gamma_{i}\right) \mathcal{P}_{\mathcal{R}_{n}} \\
& +\mathcal{P}_{\mathcal{R}_{n}} \sum_{r} \rho_{r}\left(\Gamma_{i}\right) \mathcal{P}_{\mathcal{R}_{n}} \sum_{s} \rho_{s}\left(\left[\Gamma_{i}, \quad \Gamma_{k} \Gamma_{l}\right]\right) \mathcal{P}_{\mathcal{R}_{n}} \\
& =\mathcal{P}_{\mathcal{R}_{n}} \sum_{r} \rho_{r}\left(\Gamma_{l} \delta_{i k}-\Gamma_{k} \delta_{i l}\right) \mathcal{P}_{\mathcal{R}_{n}} \sum_{s} \rho_{s}\left(\Gamma_{i}\right) \mathcal{P}_{\mathcal{R}_{n}} \\
& +\mathcal{P}_{\mathcal{R}_{n}} \sum_{r} \rho_{r}\left(\Gamma_{i}\right) \mathcal{P}_{\mathcal{R}_{n}} \sum_{s}\left(\rho_{s}\left(\Gamma_{l}\right) \delta_{i k}-\rho_{s}\left(\Gamma_{k}\right) \delta_{i l}\right) \mathcal{P}_{\mathcal{R}_{n}} \\
& =0
\end{aligned}
$$

Since $X_{i}^{2}$ commutes, we know it is a constant in each irrep.

$$
X_{i}^{2}=a_{+} \mathcal{P}_{\mathcal{R}_{n}^{+}}+a_{-} \mathcal{P}_{\mathcal{R}_{n}^{-}}
$$

Now consider the action on $X_{i}^{2}$ on $R_{+}$. We find

$$
X_{i}^{2} \mathcal{P}_{\mathcal{R}_{n}^{+}}=\mathcal{P}_{\mathcal{R}_{n}^{+}} \sum_{r} \rho_{r}\left(\Gamma_{i} P_{-}\right) \mathcal{P}_{\mathcal{R}_{n}^{-}} \sum_{s} \rho_{s}\left(\Gamma_{i} P_{+}\right) \mathcal{P}_{\mathcal{R}_{n}^{+}}=a_{+} \mathcal{P}_{\mathcal{R}_{n}^{+}}
$$


and likewise :

$$
X_{i}^{2} \mathcal{P}_{\mathcal{R}_{n}^{+}}=\mathcal{P}_{\mathcal{R}_{n}^{-}} \sum_{r} \rho_{r}\left(\Gamma_{i} P_{+}\right) \mathcal{P}_{\mathcal{R}_{n}^{+}} \sum_{s} \rho_{s}\left(\Gamma_{i} P_{-}\right) \mathcal{P}_{\mathcal{R}_{n}^{-}}=a_{-} \mathcal{P}_{\mathcal{R}_{n}^{-}}
$$

Now consider the operation of exchanging $P_{+}$with $P_{-}$, which leaves $X_{i}^{2}$ as defined in (2.3) invariant. Now peform this operation on the equation (2.7) to find

$$
X_{i}^{2} \mathcal{P}_{\mathcal{R}_{n}^{-}}=\mathcal{P}_{\mathcal{R}_{n}^{-}} \sum_{r} \rho_{r}\left(\Gamma_{i} P_{+}\right) \mathcal{P}_{\mathcal{R}_{n}^{+}} \sum_{s} \rho_{s}\left(\Gamma_{i} P_{-}\right) \mathcal{P}_{\mathcal{R}_{n}^{-}}=a_{+} \mathcal{P}_{\mathcal{R}_{n}^{-}}
$$

Comparing with (2.8) we find that $a_{+}=a_{-}$. Thus $X_{i}^{2}$ is proportional to the identity.

The radius is easily calculated as follows :

$$
X_{i}^{2} \mathcal{P}_{\mathcal{R}_{n}^{+}}=\sum_{r} \rho_{r}\left(\Gamma_{i} \Gamma_{i} P_{+}\right)+\sum_{r \neq s} \rho_{r}\left(\Gamma_{i} P_{-}\right) \rho_{s}\left(\Gamma_{i} P_{+}\right)
$$

In the second term, the index $s$ can take $\frac{n+1}{2}$ vlues for a non-zero answer and the index $r$ can take $\frac{(n-1)}{2}$ values. Also we have $\sum_{i}\left(\Gamma_{i} \otimes \Gamma_{i}\right) P_{-} \otimes P_{+}=2\left(P_{+} \otimes P_{-}\right)$. The first term gives $\frac{(n+1)}{2} \times 4$. Adding these two contributions we get, $\frac{(n+1)}{2}(4+(n-1))=\frac{(n+1)(n+3)}{2}$.

Note that

$$
\begin{aligned}
& \mathcal{P}_{\mathcal{R}_{n}} \sum_{i} \sum_{r} \rho_{r}\left(\Gamma_{i}\right) \sum_{s} \rho_{s}\left(\Gamma_{i}\right) \mathcal{P}_{\mathcal{R}_{n}} \\
& =\sum_{i} X_{i}^{2}+\mathcal{P}_{\mathcal{R}_{n}} \sum_{i} \sum_{r} \rho_{r}\left(\Gamma_{i}\right)\left(\mathcal{P}_{\mathcal{R}_{n++}}+\mathcal{P}_{\mathcal{R}_{n--}}\right) \sum_{s} \rho_{s}\left(\Gamma_{i}\right) \mathcal{P}_{\mathcal{R}_{n}}
\end{aligned}
$$

where $\mathcal{P}_{\mathcal{R}_{n++}}$ projects onto $\left(2 j_{L}, 2 j_{R}\right)=\left(\frac{n+3}{2}, \frac{n-3}{2}\right)$, and $\mathcal{P}_{\mathcal{R}_{n--}}$ projects onto $\left(2 j_{L}, 2 j_{R}\right)=$ $\left(\frac{n-3}{2}, \frac{n+3}{2}\right)$. We should be careful to distinguish the LHS of (2.11) from $X_{i}^{2}$.

The above discussion generalizes easily to fuzzy five spheres [11]. A similar calculation gives

$$
\sum_{i} X_{i}^{2}=\frac{(n+1)}{2}(n+2 k-1)
$$

\section{Fuzzy three-sphere and $S^{2} \times S^{2}$}

The Matrix Algebras related to fuzzy $S^{m}$, for $m>2$ contain more representations than required to give the algebra of functions on a sphere [11]. In the even sphere case, the higher dimensional manifolds related to the Matrix algbras have been identified [12]. We recall some relevant facts here. In the case of the even fuzzy sphere $S^{2 k}$ it was just 
$S O(2 k+1) / U(k)$. Several different proofs of this result were given. The first proof follows immediately once we know [11] that the Matrix algebra contains every representation of $S O(2 k+1)$ with unit multiplicity. We then used the result of [21] that the algebra of functions on $S O(2 k+1) / U(k)$ has precisely this property. In section 3.1 we will follow this line of argument, i.e we will compare the $S O(4)$ decomposition of the functions on $S^{2} \times S^{2}$ to the representations found in $\operatorname{End}\left(\mathcal{R}_{n}^{ \pm}\right)$at large $n$ and find agreement. In section 3.2 we will develop an analogous argument for $\operatorname{Hom}\left(\mathcal{R}_{n}^{+}, \mathcal{R}_{n}^{-}\right)$or $\operatorname{Hom}\left(\mathcal{R}_{n}^{-}, \mathcal{R}_{n}^{+}\right)$, showing that the space of sections of a bundle on $S^{2} \times S^{2}$ agrees with the $S O(4)$ content of these off-diagonal matrices. A second line of argument to relate fuzzy even spheres to the appropriate coset will be briefly described in section 3.3 and the analog will be developed for the fuzzy three-sphere.

\subsection{Spectrum of $\operatorname{End}\left(\mathcal{R}_{n}^{ \pm}\right)$and Functions on $S^{2} \times S^{2}$}

Rotations $L_{12}$ and $L_{34}$ form a $U(1) \times U(1)$ subgroup of $S O(4)$. In a self dual representation associated with an $S O(4)$ Young diagram of row lengths $\left(r_{1}, r_{2}\right)$ the eigenvalues of these generators on the highest weight vector are $r_{1}, r_{2}$. In an anti-self dual representation associated with the same Young diagram the eigenvalues on the highest weight vector are $\left(r_{1},-r_{2}\right)$. The combination $L_{12}+L_{34}$ generates a $U(1)$ subgroup of the left $S U(2)$ in the $S U(2) \times S U(2)$ description of $S O(4)$. The $S U_{L}(2)$ weight is, therefore, described by $2 J_{L}=L_{12}+L_{34}$. The combination $L_{12}-L_{34}$ generates a $U(1)$ subgroup of the right $S U(2)$. The $S U_{R}(2)$ highest weight is, therefore, described by $2 J_{R}=L_{12}-L_{34}$. For self-dual reps, we have then $2 J_{L}=r_{1}+r_{2}$ and $2 J_{R}=r_{1}-r_{2}$, where $r_{2}$ is positive. For antiself-dual reps, $2 J_{L}=r_{1}-r_{2}$ and $2 J_{R}=r_{1}+r_{2}$ for positive $r_{2}$.

To get the spectrum of representations of $S U(2) \times S U(2)$ present among the harmonics

of $\frac{(S U(2) \times S U(2))}{(U(1) \times U(1))}$ we consider the representation of $S U(2) \times S U(2)$ induced from the trivial representation of $U(1) \times U(1)$. By Frobenius duality, the multiplicity of an irrep. of $S U(2) \times S U(2)$ in the induced rep. is equal to the multiplicity of the identity rep. in the restriction of that irrep to $U(1) \times U(1)$. This allows us to recover the well-known fact that the spectrum of reps is $\left(2 j_{L}, 2 j_{R}\right)$ with $2 j_{L}$ and $2 j_{R}$ even.

Reps of highest weight $\left(r_{1}, r_{2}\right)$ with $r_{2}$ positive (i.e self-dual reps ) and $r_{1}-r_{2}$ even, is the same as the set of reps with $2 j_{L}=r_{1}+r_{2}=\left(r_{1}-r_{2}\right)+2 r_{2}$ even, and $2 j_{R}=r_{1}-r_{2}$ even, with the restriction that $2 j_{L} \geq 2 j_{R}$. Reps of highest weight $\left(r_{1},-r_{2}\right)$ with $r_{2}$ positive (i.e anti-self-dual reps ) and $r_{1}-r_{2}$ even is the same as the set described by $2 j_{L}$ even and $2 j_{R}$ even with $2 j_{R} \geq 2 j_{L}$. These sets of reps. of $S O(4)$ are the ones which appear in the 
$S O(4)$ decompositon of $\operatorname{End}\left(\mathcal{R}_{n}^{+}\right)$or $\operatorname{End}\left(\mathcal{R}_{n}^{-}\right)$at large $n$ [11]. We conclude that large $n$ $S O(4)$ decomposition of $\operatorname{End}\left(\mathcal{R}_{n}^{+}\right)$or $\operatorname{End}\left(\mathcal{R}_{n}^{+}\right)$has the same representations as the space of functions on $S^{2} \times S^{2}$. Since the structure constants of the algebra are determined by the Clebsch-Gordan coefficients, once we have established that the Matrix algebra has the same repesentations as $F u n\left(S^{2} \times S^{2}\right)$ we know that it is the same algebra.

\subsection{Spectrum of $\operatorname{Hom}\left(\mathcal{R}_{n}^{+}, \mathcal{R}_{n}^{-}\right)$and sections on $S^{2} \times S^{2}$}

In the large $n$ limit of $\operatorname{Hom}\left(\mathcal{R}_{n}^{+}, \mathcal{R}_{n}^{-}\right)\left(\right.$or $\left.\operatorname{Hom}\left(\mathcal{R}_{n}^{-}, \mathcal{R}_{n}^{+}\right)\right)$we have self-dual reps associated with Young Diagrams labelled by non-negative row lengths $\left(r_{1}, r_{2}\right)$ ( which have highest weights $\left.\left(r_{1}, r_{2}\right)\right)$, and obeying the condition $r_{1}-r_{2}$ odd. We also have antiself-dual reps. associated with Young Diagrams labelled by non-negative row lengths $\left(r_{1}, r_{2}\right)$ ( which have highest weights $\left(r_{1},-r_{2}\right)$ ), and obeying $r_{1}-r_{2}$ odd. These results are found in [11].

This approaches a space of sections on $\frac{S O(4)}{(U(1) \times U(1))}$. Spaces of sections on this coset are given by induced representations. By the the Frobenius reciprocity theorem, the multiplicity of an irrep of $R$ of $G$ in the rep. induced from $H$ using the irrep. $r$ of $H$ is equal to the multiplicity of $r$ in the restriction of $R$ to $H$.

The desired space of sections is the one obtained by inducing the irrep of $U(1) \times U(1)$ which corresponds to $(1,0)$. ( We could also work with $(-1,0)$ and get the same spectrum as $\operatorname{Hom}\left(\mathcal{R}_{n}^{-}, \mathcal{R}_{n}^{+}\right)$or $\left.\operatorname{Hom}\left(\mathcal{R}_{n}^{+}, \mathcal{R}_{n}^{-}\right)\right)$. The first $U(1)$ is generated by $L_{12}$ and the second by $L_{34}$. Writing the product of two $U(1)$ in terms of generators of the two $S U(2)$ factors in the $S O(4) \equiv S U(2) \times S U(2)$ isomorphism, the charges are $\left(2 j_{L}, 2 j_{R}\right)=(1,1)$

Consider all reps of $S U(2) \times S U(2)$ which restrict to the $\left(2 j_{L}, 2 j_{R}\right)=(1,1)$ of the $U(1) \times U(1)$ subgroup. They are all the reps with $\left(2 j_{L}, 2 j_{R}\right)$ odd and each contains the $(1,1)$ with unit multiplicity and therefore appears in the space of sections with unit multiplicity. This is exactly the description of the spectrum of $S O(4)$ reps in $\operatorname{Hom}\left(\mathcal{R}_{n}^{+}, \mathcal{R}_{n}^{-}\right)$from [11] descirbed above.

\section{3. $S^{2} \times S^{2}$ by analysis of Stabilizer group}

Another strategy in to identify the higher domensional coset in [12] was to think about the system of equations obeyed by $X_{\mu}$ and $X_{\mu \nu}$. In particular we needed to find just one solution to the system of equations, and prove that the subgroup of $S O(5)$ which kept that solution invariant was $U(2)$. To find a solution, we took the expectation value of the 
matrices $X_{\mu}$ and $X_{\mu \nu}$ in one particular state, the $n$-fold tensor product of the $v_{0}$ ( see appendix for notation ).

We will do something similar for the fuzzy 3 -sphere. Identify a set of generators for the complete Matrix algebra, and take expectation values in the large $n$ limit, and analyze the stabilizer group if the expecttaion values.

A complete set of generators is :

$$
\begin{aligned}
X_{i}^{+} & =\mathcal{P}_{\mathcal{R}_{n}^{-}} \sum_{r} \rho_{r}\left(\Gamma_{i} P_{+}\right) \mathcal{P}_{\mathcal{R}_{n}^{+}} \\
X_{i}^{-} & =\mathcal{P}_{\mathcal{R}_{n}^{+}} \sum_{r} \rho_{r}\left(\Gamma_{i} P_{-}\right) \mathcal{P}_{\mathcal{R}_{n}^{-}} \\
X_{i j}^{+} & =\mathcal{P}_{\mathcal{R}_{n}^{+}} \sum_{r} \rho_{r}\left(\frac{1}{2}\left[\Gamma_{i}, \Gamma_{j}\right] P_{+}\right) \mathcal{P}_{\mathcal{R}_{n}^{+}} \\
Y_{i j}^{+} & =\mathcal{P}_{\mathcal{R}_{n}^{+}} \sum_{r} \rho_{r}\left(\frac{1}{2}\left[\Gamma_{i}, \Gamma_{j}\right] P_{-}\right) \mathcal{P}_{\mathcal{R}_{n}^{+}} \\
X_{i j}^{-} & =\mathcal{P}_{\mathcal{R}_{n}^{-}} \sum_{r} \rho_{r}\left(\frac{1}{2}\left[\Gamma_{i}, \Gamma_{j}\right] P_{-}\right) \mathcal{P}_{\mathcal{R}_{n}^{-}} \\
Y_{i j}^{-} & =\mathcal{P}_{\mathcal{R}_{n}^{-}} \sum_{r} \rho_{r}\left(\frac{1}{2}\left[\Gamma_{i}, \Gamma_{j}\right] P_{+}\right) \mathcal{P}_{\mathcal{R}_{n}^{-}}
\end{aligned}
$$

The coordinates of the sphere $X_{i}$ are related to the above by $X_{i}=X_{i}^{+}+X_{i}^{-}$. It is also useful to define

$$
\begin{aligned}
& X_{i j}=X_{i j}^{+}+X_{i j}^{-} \\
& Y_{i j}=Y_{i j}^{+}+Y_{i j}^{-} \\
& Y_{i}=X_{i}^{+}-X_{i}^{-} \\
& \tilde{X}_{i j}=X_{i j}^{+}-X_{i j}^{-} \\
& \tilde{Y}_{i j}=Y_{i j}^{+}-Y_{i j}^{-}
\end{aligned}
$$

Take a state $\mid s>$ in $\mathcal{R}_{n}^{+}$containing $\frac{(n+1)}{2}$ copies of $v_{0}$ and $\frac{(n-1)}{2}$ copies of $a_{1}^{\dagger} v_{0}$. We find that

$$
\begin{aligned}
& <s\left|\frac{i X_{12}^{+}}{n}\right| s>=-\frac{1}{2} \\
& <s\left|\frac{i Y_{12}^{+}}{n}\right| s>=-\frac{1}{2} \\
& <s\left|\frac{i X_{34}^{+}}{n}\right| s>=-\frac{1}{2} \\
& <s\left|\frac{-i Y_{34}^{+}}{n}\right| s>=\frac{1}{2}
\end{aligned}
$$


Other expectations values are zero. The algebraic equations obeyed by $X_{i j}^{+}$and $Y_{i j}^{+}$have a solution with the above numerical values.

These values are stabilized by the rotations $L_{12}$ and $L_{34}$ which generate a $U(1) \times U(1)$ subgroup of $S O(4)$. The $S O(4)$ acts transitively on the solutions, so the set of solutions is $\frac{S O(4)}{U(1) \times U(1)}$.

\section{Summary of relations between generators}

There are the sphere-like relations which generalize the defining equation of the 3sphere.

$$
\begin{aligned}
& \sum_{i} X_{i} X_{i}=\frac{(n+1)(n+3)}{2} \\
& \sum_{i \neq j} X_{i j}^{+} X_{i j}^{+}=-(n+1)(n+5) \\
& \sum_{i \neq j} X_{i j}^{-} X_{i j}^{-}=-(n+1)(n+5) \\
& \sum_{i \neq j} Y_{i j}^{+} Y_{i j}^{+}=-(n-1)(n+3) \\
& \sum_{i \neq j} Y_{i j}^{-} Y_{i j}^{-}=-(n-1)(n+3)
\end{aligned}
$$

There are self duality or antiself-duality relations

$$
\begin{aligned}
& X_{i j}^{+}=\frac{1}{2} \epsilon_{i j k l} X_{k l}^{+} \\
& X_{i j}^{-}=-\frac{1}{2} \epsilon_{i j k l} X_{k l}^{-} \\
& Y_{i j}^{+}=-\frac{1}{2} \epsilon_{i j k l} Y_{k l}^{-} \\
& Y_{i j}^{-}=\frac{1}{2} \epsilon_{i j k l} Y_{k l}^{-}
\end{aligned}
$$

Incidentally, the (anti)self-duality equations show that there are only 3 independent components for $X_{i j}^{+}$and 3 for $Y_{i j}^{+}$. We are using conventions $\Gamma_{5}=-\Gamma_{1} \Gamma_{2} \Gamma_{3} \Gamma_{4}$ with $\Gamma_{5} v_{0}=v_{0}$. Together with the equations in (4.1) this shows explicitly the origin of the $S^{2} \times S^{2}$ in the upper block. Similarly there is the $S^{2} \times S^{2}$ in the lower block defined by the $X_{i j}^{-}$and $Y_{i j}^{-}$variables. 
Consider multiplying $X_{i}^{ \pm}$with $X_{j}^{ \pm}$

$$
\begin{aligned}
& \left(X_{i}^{+} X_{j}^{-}-X_{j}^{+} X_{i}^{-}\right)+\frac{\epsilon_{i j k l}}{2}\left(X_{k}^{+} X_{l}^{-}-X_{l}^{+} X_{k}^{-}\right)=-\frac{(n-1)}{2} \epsilon_{i j k l} X_{k l}^{-}+2\left(X_{i j}^{-}+\frac{\epsilon_{i j k l}}{2} X_{k l}^{-}\right) \\
& \left(X_{i}^{+} X_{j}^{-}-X_{j}^{+} X_{i}^{-}\right)-\frac{\epsilon_{i j k l}}{2}\left(X_{k}^{+} X_{l}^{-}-X_{l}^{+} X_{k}^{-}\right)=-\frac{n+1}{2} \epsilon_{i j k l} Y_{k l}^{-}+2\left(X_{i j}^{-}-\frac{\epsilon_{i j k l}}{2} X_{k l}^{-}\right)
\end{aligned}
$$

By converting all + to - and at the same time converting $\epsilon_{i j k l}$ to $-\epsilon_{i j k l}$, we can write the following equation :

$$
\begin{aligned}
& \left(X_{i}^{-} X_{j}^{+}-X_{j}^{-} X_{i}^{+}\right)-\frac{\epsilon_{i j k l}}{2}\left(X_{k}^{-} X_{l}^{+}-X_{l}^{-} X_{k}^{+}\right)=\frac{(n-1)}{2} \epsilon_{i j k l} X_{k l}^{+}+2\left(X_{i j}^{+}-\frac{\epsilon_{i j k l}}{2} X_{k l}^{+}\right) \\
& \left(X_{i}^{-} X_{j}^{+}-X_{j}^{-} X_{i}^{+}\right)+\frac{\epsilon_{i j k l}}{2}\left(X_{k}^{-} X_{l}^{+}-X_{l}^{-} X_{k}^{+}\right)=\frac{n+1}{2} \epsilon_{i j k l} Y_{k l}^{+}+2\left(X_{i j}^{+}+\frac{\epsilon_{i j k l}}{2} X_{k l}^{+}\right)
\end{aligned}
$$

From these we can write down

$$
\begin{aligned}
& \left(X_{i}^{-} X_{j}^{+}-X_{j}^{-} X_{i}^{+}\right)=\frac{n}{4} \epsilon_{i j k l}\left(X_{k l}^{+}+Y_{k l}^{+}\right)+\frac{1}{4} \epsilon_{i j k l}\left(-X_{k l}^{+}+Y_{k l}^{+}\right)+2 X_{i j}^{+} \\
& \left(X_{i}^{+} X_{j}^{-}-X_{j}^{+} X_{i}^{-}\right)=-\frac{n}{4} \epsilon_{i j k l}\left(X_{k l}^{-}+Y_{k l}^{-}\right)+\frac{1}{4} \epsilon_{i j k l}\left(X_{k l}^{-}-Y_{k l}^{-}\right)+2 X_{i j}^{-}
\end{aligned}
$$

By adding the two equations above we get :

$$
\begin{aligned}
{\left[X_{i}, X_{j}\right] } & =-\frac{n}{4} \epsilon_{i j k l}\left[\left(X_{k l}^{-}-X_{k l}^{+}\right)+\left(Y_{k l}^{-}-Y_{k l}^{+}\right)\right] \\
& -\frac{1}{4} \epsilon_{i j k l}\left[\left(X_{k l}^{+}-X_{k l}^{-}\right)-\left(Y_{k l}^{+}-Y_{k l}^{-}\right)\right]+2 X_{i j} \\
& =\frac{n}{4} \epsilon_{i j k l}\left(\tilde{X}_{k l}+\tilde{Y}_{k l}\right)-\frac{1}{4} \epsilon_{i j k l}\left(\tilde{X}_{k l}-\tilde{Y}_{k l}\right)+2 X_{i j}
\end{aligned}
$$

By taking the difference of the two eqs. in (4.5) we find

$$
\left\{X_{i}^{+}, X_{j}^{-}\right\}-\left\{X_{j}^{+}, X_{i}^{-}\right\}=-\frac{n}{4} \epsilon_{i j k l}\left(X_{k l}+Y_{k l}\right)+\frac{1}{4} \epsilon_{i j k l}\left(X_{k l}-Y_{k l}\right)-2\left(\tilde{X}_{i j}\right)
$$

This can be expressed as a commutator of $X_{i}$ and $Y_{i}$.

$$
\left\{X_{i}, Y_{j}\right\}=\frac{n}{4} \epsilon_{i j k l}\left(X_{k l}+Y_{k l}\right)-\frac{1}{4} \epsilon_{i j k l}\left(X_{k l}-Y_{k l}\right)+2\left(\tilde{X}_{i j}\right)
$$

We of course have relations like

$$
\begin{aligned}
& X_{i}^{+} X_{j}^{+}=0 \\
& X_{i}^{-} X_{j}^{-}=0
\end{aligned}
$$


which follow from

$$
\mathcal{P}_{\mathcal{R}_{n}^{+}} \mathcal{P}_{\mathcal{R}_{n}^{-}}=\mathcal{P}_{\mathcal{R}_{n}^{-}} \mathcal{P}_{\mathcal{R}_{n}^{+}}=0
$$

Now we consider multiplying $X_{i j}^{ \pm}$and $Y_{i j}^{ \pm}$with $X_{k}^{ \pm}$

$$
\begin{aligned}
& X_{k l}^{+} X_{i}^{-}-X_{i}^{-} Y_{k l}^{-}=\delta_{i l} X_{k}^{-}-\delta_{i k} X_{l}^{-} \\
& X_{k l}^{-} X_{i}^{+}-X_{i}^{+} Y_{k l}^{+}=\delta_{i l} X_{k}^{+}-\delta_{i k} X_{l}^{+} \\
& Y_{k l}^{-} X_{i}^{+}-X_{i}^{+} X_{k l}^{+}=\delta_{i l} X_{k}^{-}-\delta_{i k} X_{l}^{-} \\
& Y_{k l}^{+} X_{i}^{-}-X_{i}^{-} X_{k l}^{-}=\delta_{i l} X_{k}^{+}-\delta_{i k} X_{l}^{+}
\end{aligned}
$$

Defining $C_{k l i}^{j} \equiv \delta_{i l} \delta_{k j}-\delta_{i k} \delta_{j l}$ we can rewrite the above as :

$$
\begin{aligned}
X_{k l}^{+} X_{i}^{-}-X_{i}^{-} Y_{k l}^{-} & =C_{k l i}^{j} X_{j}^{-} \\
X_{k l}^{-} X_{i}^{+}-X_{i}^{+} Y_{k l}^{+} & =C_{k l i}^{j} X_{j}^{+} \\
Y_{k l}^{-} X_{i}^{+}-X_{i}^{+} X_{k l}^{+} & =C_{k l i}^{j} X_{j}^{+} \\
Y_{k l}^{+} X_{i}^{-}-X_{i}^{-} X_{k l}^{-} & =C_{k l i}^{j} X_{j}^{-}
\end{aligned}
$$

Combining these with the facts :

$$
\begin{aligned}
& X_{k l}^{+} X_{i}^{+}=X_{i}^{+} X_{k l}^{-}=0 \\
& X_{k l}^{-} X_{i}^{-}=X_{i}^{+} X_{k l}^{-}=0
\end{aligned}
$$

which follow from (4.10) these imply that $X_{k l}^{ \pm}+Y_{k l}^{ \pm}$acts by commutators as generators of $S O(4)$ rotations.

$$
\begin{aligned}
& {\left[X_{k l}^{+}+Y_{k l}^{-}, X_{i}^{-}\right]=C_{k l i}^{j} X_{j}^{-}} \\
& {\left[X_{k l}^{-}+Y_{k l}^{+}, X_{i}^{+}\right]=C_{k l i}^{j} X_{j}^{+}} \\
& {\left[X_{k l}^{+}+Y_{k l}^{-}, X_{i}^{+}\right]=C_{k l i}^{j} X_{j}^{+}} \\
& {\left[X_{k l}^{-}+Y_{k l}^{+}, X_{i}^{-}\right]=C_{k l i}^{j} X_{j}^{-}}
\end{aligned}
$$

The differences $X_{k l}^{ \pm}-Y_{k l}^{ \pm}$act by anticommutators as a rotation combined with change of $X_{i}^{+}$to $X_{i}^{-}$:

$$
\begin{aligned}
& \left\{X_{k l}^{+}-Y_{k l}^{-}, X_{i}^{-}\right\}=C_{k l i}^{j} X_{j}^{-} \\
& \left\{X_{k l}^{-}-Y_{k l}^{+}, X_{i}^{-}\right\}=-C_{k l i}^{j} X_{j}^{-} \\
& \left\{X_{k l}^{-}-Y_{k l}^{+}, X_{i}^{+}\right\}=C_{k l i}^{j} X_{j}^{+} \\
& \left\{X_{k l}^{+}-Y_{k l}^{-}, X_{i}^{+}\right\}=-C_{k l i}^{j} X_{j}^{+}
\end{aligned}
$$


Recalling from (3.2) that $X_{i}=X_{i}^{+}+X_{i}^{-}$and $Y_{i}=X_{i}^{+}-X_{i}^{-}$allows us to write some interesting anti-commutator actions :

$$
\begin{aligned}
& \left\{X_{k l}^{+}-Y_{k l}^{-}, X_{i}\right\}=-C_{k l i}^{j} Y_{j} \\
& \left\{X_{k l}^{-}-Y_{k l}^{+}, X_{i}\right\}=C_{k l i}^{j} Y_{j} \\
& \left\{X_{k l}^{+}-Y_{k l}^{-}, Y_{i}\right\}=-C_{k l i}^{j} X_{j} \\
& \left\{X_{k l}^{-}-Y_{k l}^{+}, Y_{i}\right\}=C_{k l i}^{j} X_{j}
\end{aligned}
$$

It follows that :

$$
\begin{aligned}
& {\left[X_{k l}+Y_{k l}, X_{i}\right]=2 C_{k l i}^{j} X_{j}} \\
& {\left[X_{k l}+Y_{k l}, Y_{i}\right]=2 C_{k l i}^{j} Y_{j}} \\
& \left\{X_{k l}-Y_{k l}, X_{i}\right\}=0 \\
& \left\{X_{k l}-Y_{k l}, Y_{i}\right\}=0
\end{aligned}
$$

Recalling from (3.2) that $\tilde{X}_{k l}=X_{k l}^{+}-X_{k l}^{-}$and $\tilde{Y}_{k l}=Y_{k l}^{+}-Y_{k l}^{-}$we have

$$
\begin{aligned}
& \left\{\tilde{X}_{k l}+\tilde{Y}_{k l}, X_{i}\right\}=-2 C_{k l i}^{j} Y_{j} \\
& \left\{\tilde{X}_{k l}+\tilde{Y}_{k l}, Y_{i}\right\}=-2 C_{k l i}^{j} X_{j} \\
& {\left[\tilde{X}_{k l}-\tilde{Y}_{k l}, X_{i}\right]=0} \\
& {\left[\tilde{X}_{k l}-\tilde{Y}_{k l}, Y_{i}\right]=0}
\end{aligned}
$$

So we have interesting anticommutator actions which are $S O(4)$ rotations combined with an $X-Y$ flip.

Finally we need to consider multiplication $X_{i j} X_{k l}$ and $X_{i j} Y_{k l}$ Some of these are familiar from the fuzzy $S^{4}$ case :

$$
\begin{aligned}
& {\left[X_{i j}, X_{k l}\right]=\delta_{j k} X_{i l}+\delta_{i l} X_{j k}-\delta_{j l} X_{i k}-\delta_{i l} X_{j k}} \\
& {\left[Y_{i j}, Y_{k l}\right]=\delta_{j k} Y_{i l}+\delta_{i l} Y_{j k}-\delta_{j l} Y_{i k}-\delta_{i l} Y_{j k}}
\end{aligned}
$$

Further relations are

$$
\begin{aligned}
& {\left[X_{i j}, Y_{k l}\right]=0} \\
& X_{(i j}^{+} Y_{k l)}^{+}=-\delta_{j k}\left(X_{i}^{-} X_{l}^{+}+X_{l}^{-} X_{i}^{+}\right)-\delta_{i l}\left(X_{j}^{-} X_{k}^{+}+X_{k}^{-} X_{j}^{+}\right) \\
& +\delta_{j l}\left(X_{i}^{-} X_{k}^{+}+X_{k}^{-} X_{i}^{+}\right)+\delta_{i k}\left(X_{j}^{-} X_{l}^{+}+X_{l}^{-} X_{j}^{+}\right) \\
& +\frac{(n+1)(n+3)}{2}\left(-\delta_{i k} \delta_{j l}+\delta_{i l} \delta_{j k}\right) \mathcal{P}_{\mathcal{R}_{n}^{+}} \\
& X_{i j}^{+} Y_{i l}^{+}+X_{i l}^{+} Y_{i j}^{+}=2\left(X_{j}^{-} X_{l}^{+}+X_{l}^{-} X_{j}^{+}\right)-\frac{(n+1)(n+3)}{2} \delta_{j l} \mathcal{P}_{\mathcal{R}_{n}^{+}}
\end{aligned}
$$


By taking + to - we have

$$
\begin{aligned}
& X_{(i j}^{-} Y_{k l)}^{-}=-\delta_{j k}\left(X_{i}^{+} X_{l}^{-}+X_{l}^{+} X_{i}^{-}\right)-\delta_{i l}\left(X_{j}^{+} X_{k}^{-}+X_{k}^{+} X_{j}^{-}\right) \\
& +\delta_{j l}\left(X_{i}^{+} X_{k}^{-}+X_{k}^{+} X_{i}^{-}\right)+\delta_{i k}\left(X_{j}^{+} X_{l}^{-}+X_{l}^{+} X_{j}^{-}\right) \\
& X_{i j}^{-} Y_{i l}^{-}+X_{i l}^{-} Y_{i j}^{-}=2\left(X_{j}^{+} X_{l}^{-}+X_{l}^{+} X_{j}^{-}\right)-\frac{(n+1)(n+3)}{2} \delta_{j l} \mathcal{P}_{\mathcal{R}_{n}^{-}}
\end{aligned}
$$

This implies

$$
\begin{aligned}
X_{(i j} Y_{k l)} & =\tilde{X}_{(i j}, \tilde{Y}_{k l)} \\
& =-\delta_{j k}\left\{X_{i}, X_{l}\right\}-\delta_{i l}\left\{X_{j}, X_{k}\right\}+\delta_{j l}\left\{X_{i}, X_{k}\right\}+\delta_{i k}\left\{X_{j}, X_{l}\right\} \\
& +\frac{(n+1)(n+3)}{2}\left(-\delta_{i k} \delta_{j l}+\delta_{i l} \delta_{j k}\right)
\end{aligned}
$$

A special case of (4.22) is

$$
X_{(i j} Y_{i l)}=2\left\{X_{j}, X_{l}\right\}-\frac{(n+1)(n+3)}{2} \delta_{j l}=-2\left\{Y_{j}, Y_{l}\right\}-\frac{(n+1)(n+3)}{2} \delta_{j l}
$$

It is worth pointing out that the equations of this section correct some equations in the appendix of [10] and also the remarks in section 7.3 of [11].

\section{Algebra of fuzzy 3 -sphere at large $N$}

It is useful to define, as in the case of fuzzy even spheres, rescaled variables. A surprising feature will be that, after the standard rescalings, some non-commutativity wil remain at large $n$ in the fuzzy odd cases.

We can define variables

$$
\begin{aligned}
A_{i j}^{ \pm} & =\frac{i X_{i j}^{ \pm}}{n} \\
B_{i j}^{ \pm} & =\frac{i Y_{i j}^{ \pm}}{n} \\
C_{i}^{ \pm} & =\frac{\sqrt{2} X_{i}^{ \pm}}{n}
\end{aligned}
$$

and the following linear combinations are useful.

$$
\begin{aligned}
& A_{i j}=A_{i j}^{+}+A_{i j}^{-} \\
& \tilde{A}_{i j}=A_{i j}^{+}-A_{i j}^{-} \\
& B_{i j}=B_{i j}^{+}+B_{i j}^{-} \\
& \tilde{B}_{i j}=B_{i j}^{+}-B_{i j}^{-} \\
& C_{i}=\left(C_{i}^{+}+C_{i}^{-}\right) \\
& \tilde{C}_{i}=i\left(C_{i}^{+}-C_{i}^{-}\right)
\end{aligned}
$$


The sphere-like relations are of the form :

$$
\begin{aligned}
& \sum_{i \neq j}\left(A_{i j}^{ \pm}\right)^{2}=\mathcal{P}_{\mathcal{R}_{n}^{ \pm}} \\
& \sum_{i \neq j}\left(B_{i j}^{ \pm}\right)^{2}=\mathcal{P}_{\mathcal{R}_{n}^{ \pm}} \\
& \sum_{i \neq j}\left(A_{i j}\right)^{2}=1 \\
& \sum_{i \neq j}\left(B_{i j}\right)^{2}=1 \\
& \sum_{i} C_{i}^{2}=1 \\
& \sum_{i} \tilde{C}_{i}^{2}=1
\end{aligned}
$$

We have the following vanishing commutators and anticomutators :

$$
\begin{aligned}
& {[A, A]=0} \\
& {[B, B]=0} \\
& {[A, C]=[A, \tilde{C}]=0} \\
& {[\tilde{A}+\tilde{B}, C]=[\tilde{A}+\tilde{B}, \tilde{C}]=0} \\
& \{\tilde{A}-\tilde{B}, C\}=\{\tilde{A}-\tilde{B}, \tilde{C}\}=0
\end{aligned}
$$

There are also some interesting commutators of $C, \tilde{C}$ which survive in the large $\mathrm{N}$

limit

$$
\begin{aligned}
& \left(C_{i}^{-} C_{j}^{+}-C_{j}^{-} C_{i}^{+}\right)=\frac{-i}{2} \epsilon_{i j k l}\left(A_{k l}^{+}+B_{k l}^{+}\right) \\
& \left(C_{i}^{+} C_{j}^{-}-C_{j}^{+} C_{i}^{-}\right)=\frac{i}{2} \epsilon_{i j k l}\left(A_{k l}^{-}+B_{k l}^{-}\right)
\end{aligned}
$$




$$
\begin{aligned}
{\left[C_{i}, C_{j}\right]=} & \frac{-i}{2} \epsilon_{i j k l}\left(\tilde{A}_{k l}+\tilde{B}_{k l}\right) \\
\left\{C_{i}, \tilde{C}_{j}\right\}= & \frac{-1}{2} \epsilon_{i j k l}\left(A_{k l}+B_{k l}\right) \\
A_{(i j} B_{k l)}= & -\left\{\tilde{A}_{i j}, \tilde{B}_{k l}\right\} \\
= & \frac{\delta_{j k}}{2}\left\{C_{i}, C_{l}\right\}+\frac{\delta_{i l}}{2}\left\{C_{j}, C_{k}\right\}-\frac{\delta_{j l}}{2}\left\{C_{i}, C_{k}\right\}-\frac{\delta_{i k}}{2}\left\{C_{j}, C_{l}\right\} \\
& +\frac{1}{3}\left(\delta_{i k} \delta_{j l}-\delta_{i l} \delta_{j k}\right) \\
= & +\frac{\delta_{j k}}{2}\left\{\tilde{C}_{i}, \tilde{C}_{l}\right\}+\frac{\delta_{i l}}{2}\left\{\tilde{C}_{j}, \tilde{C}_{k}\right\}-\frac{\delta_{j l}}{2}\left\{\tilde{C}_{i}, \tilde{C}_{k}\right\}-\frac{\delta_{i k}}{2}\left\{\tilde{C}_{j}, \tilde{C}_{l}\right\} \\
& +\frac{1}{3}\left(\delta_{i k} \delta_{j l}-\delta_{i l} \delta_{j k}\right) \\
A_{(i j} B_{i l)}= & -\left\{C_{j}, C_{l}\right\}+\frac{\delta_{j l}}{2}
\end{aligned}
$$

So $A, B$ should be thought of as coordinates and $C$ could perhaps be realized as derivatives. This is compatible with the observation based on group theory, that the diagonal blocks are describing functions on two copies of $S^{2} \times S^{2}$, while the off-diagonal are some sections of a bundle. The fact that within each diagonal block $\operatorname{End}\left(\mathcal{R}_{n}^{+}\right)$or $\operatorname{End}\left(\mathcal{R}_{n}^{-}\right)$ the algebra becomes commutative in the large $n$ limit, whereas there is non-commutativity in $\operatorname{End}\left(\mathcal{R}_{n}\right)$ suggests that some sort of $U(2)$ non-abelian structure is present. We will return to this in section 7 .

\section{Some Matrix Actions which admit the fuzzy three-sphere as solution}

It was observed in [14] that the fuzzy two-sphere and four sphere are solutions to Matrix brane actions which have a mass term. Such matrix models in relation to fuzzy spheres were also discussed in [22]. Mass terms also appear in the BMN matrix model and the fuzzy two-sphere appears as a supersymmetric solution of the BMN matrix model [5]. It is natural to ask if a simple Matrix Model with mass term can accommodate the fuzzy three sphere as a solution. In the case of the fuzzy even spheres the anlog of (4.6) is much simpler, since it only contains the analog of the last term on the RHS of (4.6). Further, the commutator $\left[X_{i}, X_{i j}\right]$ is proportional to $X_{j}$ in the even case, unlike the more complicated analog (4.17) which involves both $X$ and $Y$ variables. These properties of the fuzzy even spheres are adequate to prove they are solutions of a matrix model with $X_{i}$ in the vector of the $S O$ symmetry group and having a mass term. The form of the equations for the 
fuzzy three-sphere do not allow it to be a solution of such a Matrix model. It is a very interesting problem to try to find any physical Matrix model where the fuzzy three-sphere ( or the fuzzy five sphere discussed later in this paper ) is a solution. It would be even more fascinating to find these general fuzzy spheres as supersymmetry-perserving solution of any physical Matrix model. A physical Matrix model would be one that comes up as an action for branes ( parallel or intersecting ) or as a dual to some M-theory background. In the light of the difficulty of constructing a transverse five-brane from the BFSS matrix model, finding such a fuzzy five sphere as a physical Matrix model solution ( or proving it is not possible ) would give important information about the relation between M-theory and large $N$ matrix systems. We will not solve this problem in this paper but we will exhibit some toy Matrix models which do have the fuzzy three sphere as a solution, and we will observe some qualititative similarities between these toy Matrix models and some others that have appeared in recent literature.

Given the relations we wrote down in (4.6) and (4.7) it is easy to obtain some Matrix Actions which admit as solutions the matrices of the fuzzy three-sphere. Consider a Matrix action with a symmetry group which contains $S O(4))$ and consider fields transforming in the vector of $S O(4)$, labelled $\Phi_{i}$ as in the BFSS [23] or IKKT [24] 25] matrix models. Suppose, unlike these models there is an additional Matrix variable transforming in the antisymmetric of $S O(4)$ which we will denote $P_{i j}$. Suppose the action is

$$
\int \operatorname{TR}\left(\left[\Phi_{i}, \Phi_{j}\right]-P_{i j}\right)^{2}
$$

The variation of this action with respect to $\Phi_{i}$ or $P_{i j}$ will be proportional to

$$
\left[\Phi_{i}, \Phi_{j}\right]-P_{i j}
$$

From (4.6) it follows that if we set

$$
\begin{aligned}
& \Phi_{i}=X_{i} \\
& P_{i j}=\frac{n}{4} \epsilon_{i j k l}\left(\tilde{X}_{k l}+\tilde{Y}_{k l}\right)-\frac{1}{4} \epsilon_{i j k l}\left(\tilde{X}_{k l}-\tilde{Y}_{k l}\right)+2 X_{i j}
\end{aligned}
$$

where $X_{i}, X_{k l}, Y_{k l}, \tilde{X}_{k l}, \tilde{Y}_{k l}$ are defined in (3.1)(3.2), we will have a solution to the action (6.1).

Another way to construct an action which is solved by the matrices of section 3 is to start with variables $\Phi_{i}$ and $\tilde{\Phi}_{i}$ and antisymmetric Matrix variables $Q_{i j}$ which have an action

$$
\int \operatorname{TR}\left(\left\{\Phi_{i}, \tilde{\Phi}_{j}\right\}-Q_{i j}\right)^{2}
$$


In this case if we use

$$
\begin{aligned}
& \Phi_{i}=X_{i} \\
& \tilde{\Phi}_{i}=Y_{i} \\
& Q_{i j}=\frac{n}{4} \epsilon_{i j k l}\left(X_{k l}+Y_{k l}\right)-\frac{1}{4} \epsilon_{i j k l}\left(X_{k l}-Y_{k l}\right)+2\left(\tilde{X}_{i j}\right)
\end{aligned}
$$

It is interesting that in recent literature Matrix actions have been considered which involve matrices transforming in the vector of the $S O$ group as well as other antisymmetric tensors [26] 27] 28] 29]. It would be interesting to see if the fuzzy odd sphere solutions of model actions of the form (6.1) could also be found for actions involved in the above papers. Perhaps relating terms of the form in (6.1) to terms in these actions could be useful. The role of $P_{i j}$ could also be played by composite fields such as $\bar{\psi} \Gamma_{i j} \psi$.

\section{Fluctuating fields around solution}

\subsection{Physics on $S^{2} \times S^{2}$}

Useful information about the physics of a solution defined by the $X_{i}$ matrices is obtained by considering fluctuations around such a solution. In the even sphere case the action for the fluctuations is a $U(1)$ theory on $S O(2 k+1) / U(k)$ with $S O(2 k+1)$ symmetry. The existence of a hidden higher dimensional coset in the odd sphere allows a somewhat analogous result.

Imagine we find a solution to Matrix theory which uses these matrices

$$
\begin{aligned}
& \Phi_{i}=X_{i} \\
& \Phi_{a}=0
\end{aligned}
$$

The $\Phi_{i}$ transform as a vector of the $S O(4)$ symmetry group of the fuzzy three sphere, and we call them parallel scalars. The $\Phi_{a}$ are invariant under that symmetry group and are called transverse scalars.

Consider the fluctuations

$$
\begin{aligned}
& \Phi_{i}=X_{i}+\phi_{i}^{+}\left(A^{+}, B^{+}\right)+\phi_{i}^{-}\left(A^{-}, B^{-}\right)+\phi_{i j}^{+}\left(A^{+}, B^{+}\right) C_{i}^{-}+\phi_{i j}^{-}\left(A^{-}, B^{-}\right) C_{j}^{+} \\
& \Phi_{a}=\phi_{a}^{+}\left(A^{+}, B^{+}\right)+\phi_{a}^{-}\left(A^{-}, B^{-}\right)+\phi_{a i}^{+}\left(A^{+}, B^{+}\right) C_{i}^{-}+\phi_{a i}^{-}\left(A^{-}, B^{-}\right) C_{i}^{+}
\end{aligned}
$$

Each $\phi$ is a field living on $S^{2} \times S^{2}$. Notice that the transverse scalars contain components which transform as vectors under the parallel $S O(4)$. The parallel scalars give rise 
to fields $\phi_{i j}^{ \pm}$which is a two-index tensor which can be reduced to symmetric traceless part, antisymmetric part and a scalar. By expanding a Matrix action which is solved by the fuzzy three-sphere Matrices, around the solution, we would get a field theory, in analogy to the analogous results for fuzzy even spheres [30] [12] [14].

We outline some features of the field theory on $S^{2} \times S^{2}$ that results from these fluctuations. The kinetic term of $\Phi_{a}$ is

$$
\int d t \operatorname{TR}\left(\partial_{t} \Phi_{a} \partial_{t} \Phi_{a}\right)
$$

Using the expansion for $\Phi_{a}$ in (7.2), we will get, from the diagonal terms,

$$
\begin{aligned}
& \int d t d A^{+} d B^{+}\left(\partial_{t} \phi_{a}^{+}\left(A^{+}, B^{+}\right)\right)^{2}+\int d t d A^{-} d B^{-}\left(\partial_{t} \phi_{a}^{-}\left(A^{-}, B^{-}\right)\right)^{2} \\
& =\int d t d A d B\left(\partial_{t} \phi_{a}^{+}(A, B)\right)^{2}+\left(\partial_{t} \phi_{a}^{-}(A, B)\right)^{2}
\end{aligned}
$$

$d A^{+} d B^{+}=d A^{-} d B^{-}$is an $S O(4)$ invariant measure for $S^{2} \times S^{2}$. These diagonal terms coming from the large $n$ limit of $\operatorname{End}\left(\mathcal{R}_{n}^{+}\right)$and $\operatorname{End}\left(\mathcal{R}_{n}^{-}\right)$. From squaring the off diagonal terms, $\phi_{a i}^{+}\left(A^{+}, B^{+}\right) C_{i}^{-}+\phi_{a i}^{-}\left(A^{-}, B^{-}\right) C_{i}^{+}$we obtain terms including

$$
\int d t T R_{\mathcal{R}_{n}^{+}}\left(\partial_{t} \phi_{a i}^{+}\left(A^{+}, B^{+}\right) C_{i}^{-} \partial_{t} \phi_{a i}^{-}\left(A^{-}, B^{-}\right) C_{j}^{+}\right)
$$

After rescalings of (5.1) the relations in (4.12) allow us to show that polynomials in $A^{-}, B^{-}$ can be pulled to the left of $C_{i}^{-}$at the cost of converting the pair $\left(A^{-}, B^{-}\right)$to $\left(B^{+}, A^{+}\right)$ ( not the switch). There are extra terms coming from the RHS of (4.12) which can be ignored in the leading large $n$ limit. The terms in (7.5) can then be written as

$$
\int d t T R_{\mathcal{R}_{n}^{+}}\left(\partial_{t} \phi_{a i}^{+}\left(A^{+}, B^{+}\right) \partial_{t} \phi_{a j}^{-}\left(B^{+}, A^{+}\right) C_{i}^{-} C_{j}^{+}\right)
$$

It is useful to write this as a product of parts symmetric in $(i j)$ and a part antisymmetric in these indices. The symmetric part is simplified using (4.20) to replace

$$
\left(C_{i}^{-} C_{j}^{+}+C_{j}^{-} C_{i}^{+}\right)=\frac{1}{2} \delta_{i j}-A_{k i}^{+} B_{k j}^{+}-A_{k j}^{+} B_{k i}^{+} \equiv G_{i j}
$$

The antisymmetric part is simplified using (4.5)

$$
\left(C_{i}^{-} C_{j}^{+}-C_{j}^{-} C_{i}^{+}\right)=\frac{-i}{2} \epsilon_{i j k l}\left(A_{k l}^{+}+B_{k l}^{+}\right) \equiv H_{i j}
$$


We have defined in (7.7) and (7.8) a symmetric tensor and an antisymmetric tensor living on $S^{2} \times S^{2}$. After converting the trace to an integral using $S O(4)$ invariance, we can write the kinetic terms in (7.5) as

$$
\int d t \int d A d B \partial_{t} \phi_{a i}^{+}(A, B) \partial_{t} \phi_{a j}^{-}(B, A)\left(G_{i j}+H_{i j}\right)
$$

Analogous to the term in (7.5) there is a trace over $\mathcal{R}_{n}^{-}$which leads by similar steps as above to

$$
\int d t \int d A d B \partial_{t} \phi_{a i}^{-}(A, B) \partial_{t} \phi_{a j}^{+}(B, A)\left(G_{i j}-H_{i j}\right)
$$

Although $H$ contains $i$ the action is hermitian since hermitian conjugation converts $\phi^{+}$to $\phi^{-}$.

The above arguments illustrate the use of the algebraic relations of section 4 in deriving the action for fluctuations. It appears that there is some $U(2)$ symmetry which mixes the $\mathcal{R}_{n}^{+}$and the $\mathcal{R}_{n}^{-}$but which is broken to $U(1) \times U(1)$. We leave it to the future to elucidate all the symmetries of such an action. A further interesting problem is to relate this theory on $S^{2} \times S^{2}$ to some field theory on $S^{3}$. In the fuzzy even sphere case there was a $U(1)$ theory on the higher dimensional geometry and a non-abelian theory on the sphere itself [12]. We would expect a generalization of that correspondence to yield some field theory on the sphere $S^{3}$. The counting of degrees of freedom in section 9 shows that it cannot be a standard non-abelian theory. The discussion in section 9 will also show that the analogous field theory on the coset $\frac{S O(6)}{U(2) \times U(1)}$ might be more easily related to a field theory on the five sphere.

\section{The fuzzy five-sphere}

\subsection{Higher dimensional geometry from Stabilizer group}

A guess is that $\operatorname{End}\left(\mathcal{R}_{n}^{+}\right)$related to $\frac{S O(2 k)}{U(k-1) \times U(1)}$. This works in the case of $k=2$, and gives the right counting of degrees of freedom, i.e $N^{2}$ scales like $n^{10}$ while quadratic expressions like $X_{i}^{2}$ scale like $n^{2}$. This means we should expect a 10 dimensional space which is indeed the dimension of the above coset for $k=3$. Here we will develop an argument similar to section 3.3 based on the expectation values of generators of the matrix algebra evaluated in a state in $\mathcal{R}_{n}$. We pick a state of the form $v_{0} \otimes \cdots \otimes v_{0} \otimes a_{1}^{\dagger} v_{0} \cdots a_{1}^{\dagger} v_{0}$, where there are $\frac{(n+1)}{2}$ copies of $v_{0}$ and $\frac{(n-1)}{2}$ copies of $a_{1}^{\dagger} v_{0}$. We sum over different ways 
of embedding the copies $v_{0}$ and the $a_{1}^{\dagger} v_{0}$ in $V^{\otimes n}$ in order to make sure we have a state in $\operatorname{Sym}\left(V^{\otimes n}\right)$.

Considering normalized quantities analogous to (5.1), we have in the large $n$ limit

$$
\begin{aligned}
& <s\left|A_{12}^{+}\right| s>=1 \\
& <s\left|A_{34}^{+}\right| s>=<s\left|A_{56}^{+}\right| s>=1 \\
& <s\left|C_{1}^{2}\right| s>=1 / 2 \\
& <s\left|C_{2}^{2}\right| s>=1 / 2 \\
& <s\left|C_{1} C_{2}+C_{2} C_{1}\right| s>=0 \\
& <s\left|C_{1} C_{3}+C_{3} C_{1}\right| s>=0 \ldots \\
& <s\left|B_{12}^{+}\right| s>=-1 \\
& <s\left|B_{34}^{+}\right| s>=<s\left|B_{56}^{+}\right| s>=1
\end{aligned}
$$

Other $A_{i j}$ and $B_{i j}$ have zero expectation value. The above variables are defined in the same way as for the fuzzy 3-sphere. These expectation values in (8.1) are only preseved by $U(1) \times U(2)$. This means that the complete set of variables describing the matrix algebra $\operatorname{End}\left(\mathcal{R}_{n}^{+}\right)$in the large $n$ limit have a solution which is stabilized by the subgroup $U(1) \times U(2)$. The full set of solutions, obtained by action of $S O(6)$ on the irrep $\mathcal{R}_{n}^{+}$, is acted on transitively by the $S O(6)$. Hence the set of solutions is the coset $S O(6) / U(2) \times U(1)$. The $U(1)$ is generated by rotations in the 12 direction. In the 3546 block there is a $U(2)$ subgroup which preserves the expectation values. Consider $\left.<X_{1}^{2}\right\rangle=<X_{2}^{2}>=1$. These are matrices of the form

$$
\left(\begin{array}{cc}
P & Q \\
-Q & P
\end{array}\right)
$$

where $P$ is real antisymetric and $Q$ is real symmtric.

\subsection{Group Theory Proof: Harmonics of $S O(6) / U(2) \times U(1)$ from Matrix realization}

In the next two sections, we develop the arguments analogous to sections 3.1 and 3.2, now for the case of the fuzzy five-sphere. We recall from [11 the list of representations of $S O(6)$ which appear in $\operatorname{End}\left(\mathcal{R}_{n}^{+}\right)$at large $n$. There are self-dual representations associated with highest weights $\vec{\lambda}=\left(p_{1}+p_{2}+p_{3}, p_{1}+p_{2}, p_{1}\right)$, and antiself-dual reps with highest weights $\vec{\lambda}=\left(p_{1}+p_{2}+p_{3}, p_{1}+p_{2},-p_{1}\right)$, where $p_{1}, p_{2}, p_{3}$ are positive. The associated Young diagrams have row lengths $\vec{r}=\left(p_{1}+p_{2}+p_{3}, p_{1}+p_{2}, p_{1}\right)$ Unlike the case of the fuzzy three sphere, we now have representations with multiplicity more than 2 . The multiplicity is 
zero unless the following conditions are satisfied: $p_{1}+p_{3}$ is even, if $p_{1}$ is positive, $p_{3} \geq p_{1}$ and if $p_{1}$ is negative, $p_{3} \geq\left|p_{1}\right|$. Then the multiplicity is

$$
m\left(p_{1}, p_{2}, p_{3}\right)=p_{2}+1
$$

The multiplicity arises because of the multiple ways of writing $p_{2}$ as a sum of two nonnegative integers $p_{2}^{+}$and $p_{2}^{-}$. Different choices of $p_{2}^{+}$and $p_{2}^{-}$lead to different operators in $\operatorname{End}\left(\mathcal{R}_{n}^{+}\right)$which transform according to the same representation of $S O(6)$.

The space of functions on $\frac{S O(6)}{U(2) \times U(1)}$ is the $S O(6)$ representation induced from the trivial rep. of $U(2) \times U(1)$. By Frobenius duality, multiplicity of an $S O(6)$ irrep. in the induced rep is the same as the multiplicity of the trivial rep. of $U(2) \times U(1)$ in that $S O(6)$ representation [19]. We will compare, therefore, the multiplicity of the trivial rep. of $U(1) \times U(2)$ in the restriction of any given $S O(6)$ irreducible representation with the number of times that irrep. appears in $\operatorname{End}\left(\mathcal{R}_{n}^{+}\right)$. We expect they are equal.

We saw in 8.2 that the multiplicities in $\operatorname{End}\left(\mathcal{R}_{n}^{+}\right)$are zero unless $p_{1}+p_{3}$ is even. This can be shown easily to be a property of the restriction multiplicities as well, from facts about the root lattice of $S O(6)$ [31]. We show that unless $p_{1}+p_{3}$ is an even integer, the multiplicity of appearance of the trivial rep. of $U(2) \times U(1)$ is zero. A necessary condition for the trivial rep. to appear is that the multiplicity of the zero weight vector is non-zero. Unless $p_{1}+p_{3}$ is even this multiplicity is actually zero.

Indeed, the positive roots are $L_{1} \pm L_{2}, L_{2} \pm L_{3}, L_{1} \pm L_{3}$ [31]. The highest weight of the irrep. characterized by $\vec{\lambda}=\left(p_{1}+p_{2}+p_{3}, p_{1}+p_{2}, p_{1}\right)$ is $\left(p_{1}+p_{2}+p_{3}\right)\left(L_{1}\right)+\left(p_{1}+\right.$ $\left.p_{2}\right)\left(L_{1}+L_{2}\right)+\left(p_{1}\right)\left(L_{1}+L_{2}+L_{3}\right)$. If any state of weight zero exists in the rep. it must be obtained by subtracting from the highest weight a positive root. So we need to write

$$
\vec{\lambda}=a_{1}\left(L_{1}+L_{2}\right)+a_{2}\left(L_{1}-L_{2}\right)+a_{3}\left(L_{2}+L_{3}\right)+a_{4}\left(L_{2}-L_{3}\right)+a_{5}\left(L_{1}+L_{3}\right)+a_{6}\left(L_{1}-L_{3}\right)
$$

If we add up the components of $\vec{\lambda}$ we get $2 p_{2}+3 p_{1}+p_{3}$ which has to equal $2 a_{1}+2 a_{2}+$ $2 a_{3}+2 a_{4}+2 a_{5}+2 a_{6}$. Since the $a_{i}$ are positive integers, it is clear that this is only possible if $p_{1}+p_{3}$ is even.

In the case where $p_{1}+p_{3}$ is even but $p_{3}<p_{1}$ the multiplicity of the identity rep. of $U(1) \times U(2)$ is zero. The identity rep. is characterized by having a state of weight zero, which is annihilated by the raising or lowering operators of the $U(2)$. In the case $p_{1}+p_{3}$ odd, the multiplicity of the zero weight vector is zero, so it is clear that there is no trivial 
rep. In the case where $p_{1}+p_{3}$ is even but $p_{3}<p_{1}$, the multiplicity of the zero weight vector is non-zero, but it belongs to a higher rep. of $U(1) \times U(2)$.

We need to recall some details about the embedding of $U(1) \times U(2)$. Let $H_{1}, H_{2}, H_{3}$ be the generators of the Cartan of $S O(6) . H_{1}=L_{12}, H_{2}=L_{34}, H_{3}=L_{56}$. The $U(1)$ appearing as a factor in the subgroup is generated by $H_{1}$. The abelian $U(1)$ of the $U(2)$ is generated by $H_{2}-H_{3}$. The Cartan of the $S U(2)$ subgroup of the $U(2)$ is $H_{2}+H_{3}$. The trivial rep. of $U(1) \times U(2)$ has $\left(H_{1}, H_{2}, H_{3}\right)=(0,0,0)$. Non-trivial reps. of this subgroup which also contain the zero vector have highest weights of the form $(0, k,-k)$. Let $m(0, k,-k)$ be the multiplicity of the state of weight $(0, k,-k)$. Let $m_{h}(0, k,-k)$ be the multiplicity of $(0, k,-k)$ as a highest weight. For a fixed rep. of $S O(6)$ let $(0, K,-K)$ be the highest $U(1) \times U(2)$ weight which appears in the restriction to $U(1) \times U(2)$. The problem of finding the multiplicity of the trivial rep. is then reduced to calculating multiplicities of such states. Indeed

$$
\begin{aligned}
& m_{h}(0,0,0)=m(0,0,0)-m_{h}(0,0,0)-m_{h}(0,1,-1)-\cdots m_{h}(0, K,-K) \\
& m_{h}(0,1,-1)=m(0,1,-1)-m_{h}(0,2,-2)-\cdots m_{h}(0, K,-K) \\
& m_{h}(0,2,-2)=m(0,2,-2)-m_{h}(0,3,-3)-\cdots m_{h}(0, K,-K) \\
& \vdots \\
& m_{h}(0, K,-K)=m(0, K,-K)
\end{aligned}
$$

From these equations it follows that

$$
m_{h}(0,0,0)=m(0,0,0)-m(0,1,-1)
$$

In the table below we give the relevant multiplicities for some of irreps. of $S O(6)$ and we calculate $m_{h}(0,0,0)$. The multiplicity obtained from the Matrix decomposition has been expressed in the final column using a $\theta$ function to express the fact that it is non-zero only when $p_{3}>\left|p_{1}\right|$ The multiplicities of the weight vectors are calculated on Maple using the Weyl character formula for $S O(6)$ ( see for example [31] ). We observe that the multiplicity calculated using the character formula is equal to that we expect from the Matrix Decomposition, as shown by the agreement of the last two columns of the table.

We have also performed the above checks when $p_{1}$ is negative, i.e the reps are antiself-dual, and we again get agreement between the Matrix decomposition multiplicities 


\begin{tabular}{|c|c|c|c|c|}
\hline$\vec{\lambda}$ & $m(0,0,0)$ & $m(0,1,-1)$ & $m_{h}(0,0,0)$ & $\left(p_{2}+1\right) \Theta\left(p_{3}-p_{1}\right)$ \\
\hline$(2,0,0)$ & 2 & 1 & 1 & 1 \\
\hline$(4,0,0)$ & 3 & 2 & 1 & 1 \\
\hline$(6,0,0)$ & 4 & 3 & 1 & 1 \\
\hline$(8,0,0)$ & 5 & 4 & 1 & 1 \\
\hline$(1,1,0)$ & 3 & 1 & 2 & 2 \\
\hline$(3,1,0)$ & 7 & 5 & 2 & 2 \\
\hline$(5,1,0)$ & 11 & 9 & 2 & 2 \\
\hline$(2,2,0)$ & 6 & 3 & 3 & 3 \\
\hline$(4,2,0)$ & 15 & 12 & 3 & 3 \\
\hline$(6,2,0)$ & 24 & 21 & 3 & 3 \\
\hline$(3,3,0)$ & 10 & 6 & 4 & 4 \\
\hline$(5,3,0)$ & 26 & 22 & 4 & 4 \\
\hline$(7,3,0)$ & 42 & 38 & 4 & 4 \\
\hline$(4,4,0)$ & 15 & 10 & 5 & 5 \\
\hline$(6,4,0)$ & 40 & 35 & 5 & 5 \\
\hline$(5,5,0)$ & 21 & 15 & 6 & 6 \\
\hline$(2,1,1)$ & 3 & 2 & 1 & 1 \\
\hline$(4,1,1)$ & 6 & 5 & 1 & 1 \\
\hline$(6,1,1)$ & 9 & 8 & 1 & 1 \\
\hline$(3,2,1)$ & 8 & 6 & 2 & 2 \\
\hline$(5,2,1)$ & 16 & 14 & 2 & 2 \\
\hline$(7,2,1)$ & 24 & 22 & 2 & 2 \\
\hline$(4,3,1)$ & 15 & 12 & 3 & 3 \\
\hline$(6,3,1)$ & 30 & 27 & 3 & 3 \\
\hline$(5,4,1)$ & 24 & 20 & 4 & 4 \\
\hline$(4,2,2)$ & 6 & 5 & 1 & 1 \\
\hline$(6,2,2)$ & 11 & 10 & 1 & 1 \\
\hline$(5,3,2)$ & 15 & 13 & 2 & 2 \\
\hline$(4,4,2)$ & 6 & 6 & 0 & 0 \\
\hline$(4,3,3)$ & 3 & 3 & 0 & 0 \\
\hline$(6,3,3)$ & 10 & 9 & 1 & 1 \\
\hline$(7,4,3)$ & 24 & 22 & 1 & 1 \\
\hline
\end{tabular}

and those obtained from counting weights. It would be interesting to develop this proof using characters into an analytic proof but we will leave that to the enthusiastic reader, 
and hope the remaining readers will be content with the agreement demonstrated in the table below.

\section{3. $\operatorname{Hom}\left(\mathcal{R}_{n}^{+}, \mathcal{R}_{n}^{-}\right)$as sections}

We have matched the matrices which map $\mathcal{R}_{n}^{+}$to $\mathcal{R}_{n}^{+}$, i.e endomorphisms $\operatorname{End}\left(\mathcal{R}_{n}^{+}, \mathcal{R}_{n}^{+}\right)$to functions on the coset $\frac{S O(6)}{U(2) \times U(1)}$. The matrices $\operatorname{End}\left(\mathcal{R}_{n}^{-}, \mathcal{R}_{n}^{-}\right)$have the same decomposition into $S O(6)$ representations as $\operatorname{End}\left(\mathcal{R}_{n}^{+}, \mathcal{R}_{n}^{+}\right)$. The matrices which map $\mathcal{R}_{n}^{+}$to $\mathcal{R}^{-}$, or homomorphisms $\operatorname{Hom}\left(\mathcal{R}_{n}^{+}, \mathcal{R}_{n}^{-}\right)$have a different $S O(6)$ decomposition. Here we will show that the $S O(6)$ decomposition of $\operatorname{Hom}\left(\mathcal{R}_{n}^{+}, \mathcal{R}_{n}^{-}\right)$matches that of sections of a certain bundle over $\frac{S O(6)}{U(2) \times U(1)}$.

Sections of bundles over a coset $G / H$ are induced representations. They are specified by choosing a representation $R_{H}$ of the subgroup $H$ constructing the representation of $G$ induced from it. In the discussion above, $R_{H}$ was the trivial bundle. By the Frobenius reciprocity theorem, the multiplicity of an irreducible representation of $R_{G}$ of $G$ in the induced representation is equal to the multiplicity of $R_{H}$ in the restriction of $R_{G}$ to $H$. Sections of a non-trivial bundle are obtained by chooosing a non-trivial representation of $H$. Here we show that $\operatorname{Hom}\left(\mathcal{R}_{n}^{+}, \mathcal{R}_{n}^{-}\right)$matches the representation of $S O(6)$ induced from $(1,0,0)$ of $U(1) \times U(2)$. The first integer here is the $U(1)$ charge and the last two integers label the representation of $U(2)$. $\operatorname{Hom}\left(\mathcal{R}_{n}^{-}, \mathcal{R}_{n}^{+}\right)$matches the representation of $S O(6)$ induced from $(-1,0,0)$ of $U(1) \times U(2)$.

We will give the evidence in the case of $\operatorname{Hom}\left(\mathcal{R}_{n}^{+}, \mathcal{R}_{n}^{-}\right)$. Let us recall from [1] the multiplicities of irreps. of $S O(6)$ appearing in $\operatorname{Hom}\left(\mathcal{R}_{n}^{+}, \mathcal{R}_{n}^{-}\right)$. For positive integers $p_{1}, p_{2}, p_{3}$ there are self-dual representations $\left(\lambda_{1}, \lambda_{2}, \lambda_{3}\right)=\left(p_{1}+p_{2}+p_{3}, p_{1}+p_{2}, p_{1}\right)$. The multiplicities are $m(\lambda)=p_{2}+1$ if $p_{1}+p_{3}$ is odd and $p_{3} \geq p_{1}-1$ If these conditions on $p_{1}$ and $p_{3}$ are not satisfied, then the multiplicity is zero. At finite $n$ there is an upper limit $p_{1}+p_{2}+p_{3} \leq n$, but as $n \rightarrow \infty$ there is no upper limit. There are also anti-selfdual representations labelled by $\left(\lambda_{1}, \lambda_{2}, \lambda_{3}\right)=\left(p_{1}+p_{2}+p_{3}, p_{1}+p_{2},-p_{1}\right)$, so that $\lambda_{3}$ is negative, while $p_{1}, p_{2}, p_{3}$ are still positive. Again the condition for a non-zero multiplicity is that $p_{1}+p_{3}$ is odd, and that $p_{3} \geq p_{1}+1$. When these conditions are satisfied, the multiplicity is given by $m(\vec{\lambda})=p_{2}+1$. These multiplicities from the Matrix deomposition have to agree with restriction multiplicities, i.e the number of times $(1,0,0)$ appears as a $U(2) \times U(1)$ highest weight for any given highest weight $\vec{\lambda}$ of $S O(6)$.

The condition $p_{1}+p_{3}$ be odd is easy to prove ( just as the evenness of $p_{1}+p_{3}$ is easy to prove in the case of restriction multiplicities for the trivial rep. of $S O(6))$. It simply 
follows from the fact that if $p_{1}+p_{3}$ is even, the corresponding highest weight differs from the vector $(1,0,0)$ by an element which is not in the root lattice. This means that the multiplicity of $(1,0,0)$ is zero. In the case where $p_{1}+p_{3}$ is odd but the inequalities between $p_{1}$ and $p_{3}$ are not respected, the multiplicity of the weight vector $(1,0,0)$ is non-zero, but its multiplicity as a highest weight of $U(1) \times U(2)$ is zero.

\begin{tabular}{|c|c|c|c|c|}
\hline$\vec{\lambda}$ & $m(1,0,0)$ & $m(1,1,-1)$ & $m_{h}(1,0,0)$ & $\left(p_{2}+1\right) \Theta\left(p_{3}-p_{1}+1\right)$ \\
\hline$(1,0,0)$ & 1 & 0 & 1 & 1 \\
\hline$(3,0,0)$ & 2 & 1 & 1 & 1 \\
\hline$(5,0,0)$ & 3 & 2 & 1 & 1 \\
\hline$(7,0,0)$ & 4 & 3 & 1 & 1 \\
\hline$(2,1,0)$ & 4 & 2 & 2 & 2 \\
\hline$(4,1,0)$ & 8 & 6 & 2 & 2 \\
\hline$(6,1,0)$ & 12 & 10 & 2 & 2 \\
\hline$(8,1,0)$ & 16 & 14 & 2 & 2 \\
\hline$(3,2,0)$ & 9 & 6 & 3 & 3 \\
\hline$(5,2,0)$ & 18 & 15 & 3 & 3 \\
\hline$(7,2,0)$ & 27 & 24 & 3 & 3 \\
\hline$(4,3,0)$ & 16 & 12 & 4 & 4 \\
\hline$(6,3,0)$ & 32 & 28 & 4 & 4 \\
\hline$(5,4,0)$ & 25 & 20 & 5 & 5 \\
\hline$(3,1,1)$ & 4 & 3 & 1 & 1 \\
\hline$(5,1,1)$ & 7 & 6 & 1 & 1 \\
\hline$(7,1,1)$ & 10 & 9 & 1 & 1 \\
\hline$(5,2,2)$ & 8 & 7 & 1 & 1 \\
\hline$(7,2,2)$ & 13 & 12 & 1 & 1 \\
\hline$(4,3,2)$ & 8 & 6 & 2 & 2 \\
\hline$(6,3,2)$ & 20 & 18 & 2 & 2 \\
\hline$(3,3,3)$ & 1 & 1 & 0 & 0 \\
\hline$(5,3,3)$ & 6 & 5 & 1 & 1 \\
\hline$(5,4,4)$ & 3 & 3 & 0 & 0 \\
\hline$(7,4,4)$ & 10 & 9 & 1 & 1 \\
\hline
\end{tabular}

The multiplicity of $(1,0,0)$ as a highest weight of $U(1) \times U(2)$, which we denote as $m_{h}(1,0,0)$, is related to the multiplicities of weights of the form $(1,0,0),(1,1,-1), \cdots$ $(1, K,-K)$ where $(1, K,-K)$ is the highest weight of this form appearing in the irrep. 
specified by the highest weight $\vec{\lambda} . m(1,0,0)$ and $m(1,1,-1)$ by

$$
\begin{aligned}
& m_{h}(1,0,0)=m(1,0,0)-\left(m_{h}(1,1,-1)+\cdots+m_{h}(1, K,-K)\right) \\
& m_{h}(1,1,-1)=m(1,1,-1)-\left(m_{h}(1,1,-1)+\cdots m_{h}(1, K,-K)\right) \\
& \vdots \\
& m_{h}(1,2,-2)=m(1,2,-2)-\left(m_{h}(1,2,-2)+\cdots m(1, K,-K)\right) \\
& m_{h}(1, K,-K)=m(1, K,-K)
\end{aligned}
$$

which leads to a simple equation for $m_{h}(1,0,0)$ in terms of $m(1,0,0)$ and $m(1,1,-1)$.

$$
m_{h}(1,0,0)=m(1,0,0)-m(1,1,-1)
$$

The table above gives a counting of weight multiplicities performed with Maple using the Weyl character formula for $S O(6)$ and shows that the counting of multiplicities of the $U(2) \times U(1)$ irrep. obtained by restriction of $S O(6)$ agrees with that obtained from analysing the $S O(6)$ content of $\operatorname{Hom}\left(\mathcal{R}_{n}^{+} \cdot \mathcal{R}_{n}^{-}\right)$.

\section{Some remarks on geometry and combinatorics of fuzzy odd spheres}

\section{1. geometry of fuzzy $S^{2 k}$}

The coset $\frac{S O(2 k)}{U(k-1) \times U(1)}$ can be seen to admit the following bundle structure,

$$
\begin{aligned}
& \frac{S O(2 k)}{U(k-1) \times U(1)} \longleftarrow \frac{U(k)}{U(k-1) \times U(1)} \\
& \downarrow \\
& \frac{S O(2 k)}{U(k)}
\end{aligned}
$$

using the general bundle structure $H / K \rightarrow G / K \rightarrow G / H$ which exists whwnever we have subgroups $K \subset H \subset G$. The base is a hermitian symmetric space ( incidentally one of the kind that comes up as the fibre of the bundle structure exploited in 12 for fuzzy even spheres). The fibre here is actually a coset description of $C P^{k-1}$, also a symmetric space. In the case $k=2$, the base and fibre are both $S^{2}$. In the case $k=3$, the base is $C P^{3}$ and the fibre $C P^{2}$. In the case $k=4$, the fibre is $C P^{4}$ and the base is $S O(8) / U(4)$.

In the case $k=2$, the base $S^{2}$ is of lower dimension than the sphere $S^{3}$ we started with. For higher $k$ the base of the above fibration is always of higher dimension than 
the odd sphere $S^{2 k-1}$. The case $k=3$ of the fuzzy five sphere is rather special in that the $C P^{3}$ is simply related to the $S^{5}$ by a $U(1)$ quotient, $S^{5}=C P^{3} / U(1)$, a fact which found a recent physical application in [32] for example. In [12] the bundle structure of the higher dimensional geometry was used to obtain a non-abelian theory on the sphere. It is possible that the special relation of $C P^{3}$ to $S^{5}$ will allow the Matrix algebra related to fuzzy 5 -sphere to be related to field theory on 5 -sphere, and hence to brane physics of spherical branes, more easily than the other cases. It is intriguing that the physics of type IIA theory which contains both supersymmetric zero-branes and NS-5 branes might also be taken to suggest, using a simple-minded asumption that zero-branes should in some context blow-up into NS-5, that fuzzy 5-spheres would be easier than other odd spheres.

Another possibly useful bundle structure is

$$
\begin{aligned}
& \frac{S O(2 k)}{U(k-1) \times U(1)} \longleftarrow \frac{S O(2 k-2) \times U(1)}{U(k-1) \times U(1)}=\frac{S O(2 k-2)}{U(k-1)} \\
& \quad \downarrow \\
& \frac{S O(2 k)}{U(1) \times S O(2 k-2)}
\end{aligned}
$$

\subsection{Projecting to $S^{2 k-1}$ and possible role for $S^{2 k-1} / Z_{2}=R P^{2 k-1}$}

In the case of the fuzzy even sphere, there is a simple way to project the Matrix algebra onto the $S O(2 k+1)$ reps which are symmetric traceless, and which approach the algebra of functions on a sphere at large $n$. This gives rise to a non-associative algebra at finite $n[11]$ [12]. We can apply a projection to get a space of functions which is the same as the space of functions on $S^{2 k-1}$. This requires the off diagonal components. If we only keep Matrices which transform as symmetric traceless irreps. of $S O(2 k-1)$ and and which are invariant under conjugation by a permutation which switches positive chirality with negative, this will give us the desired representations. This projects out the $Y_{i}$, while keeping $X_{i}$ and their symmetric products. It also kills off $X_{i j}^{ \pm}, Y_{i j}^{ \pm}$

If we look at the upper block alone $\operatorname{End}\left(\mathcal{R}_{n}^{+}\right)$and project out antisymmetric representations we are left with the algebra of functions on $R P^{2 k-1}=S^{2 k-1} / Z_{2}$. The matrices transforming as a vector, i.e the $X_{i}$, are projected out as well as those which transforming according to any $S O(2 k)$ Young diagram with $\vec{r}=\left(r_{1}, 0 \cdots\right)$ and $r_{1}$ odd. The advantage of working with $R P^{2 k-1}$ is that in the large $n$ limit, the algebra becomes associative as in the case of the algebra of the fuzzy four-sphere and for similar reasons. The correlation between spatial $S O(2 k)$ transformation properties and a $Z_{2}$ subgroup of a $U(2)$ is reminiscent of structures encountered in the study of zero branes on compact orbifolds in [33] [34]. These analogies may be useful in finding physical brane realizations of fuzzy odd spheres. 


\subsection{Combinatoric comparison of even and odd spheres}

In the even fuzzy sphere cases we can deduce the rank of the non-abelian group by looking at the geometry. The radius always scales as $n$. The number of degrees of freedom ( dof ) required to describe the sphere scales as $n^{2 k}$ for $S^{2 k}$. The total number of dof scales as $N^{2}$, where $N$ is the size of the matrix, i.e the dimension of $\mathcal{R}_{n}$. It is also equal to $n^{D}$ where $D$ is the dimension of the higher dimensional coset. When we formulate the physics as a non-abelian one, the rank is $n^{\frac{D-2 k}{2}}$. This is made concrete in the table below.

\begin{tabular}{|c|c|c|c|c|c|}
\hline $\begin{array}{c}\text { Fuzzy sphere } \\
S^{D}\end{array}$ & $\begin{array}{c}n \text {-scaling of } \\
\text { Radius } \\
D=2 k\end{array}$ & $\begin{array}{c}n \text {-scaling of } \\
\text { geometrical } \\
\text { dof } n^{D}\end{array}$ & $\begin{array}{c}n \text {-scaling of } \\
\text { total dof } N^{2} \sim n^{T} \\
=n^{k^{2}+k}\end{array}$ & $\begin{array}{c}\text { n-scaling of } \\
\text { excess dof } \\
n^{T-D}\end{array}$ & $\begin{array}{c}n \text {-scaling for rank } \\
\text { of gauge } \\
\text { group }=n^{\frac{T-D}{2}}\end{array}$ \\
\hline$S^{2}$ & $n$ & $n^{2}$ & $n^{2}$ & 1 & 1 \\
\hline$S^{4}$ & $n$ & $n^{4}$ & $n^{6}$ & $n^{2}$ & $n$ \\
\hline$S^{6}$ & $n$ & $n^{6}$ & $n^{12}$ & $n^{6}$ & $n^{3}$ \\
\hline$S^{8}$ & $n$ & $n^{8}$ & $n^{20}$ & $n^{12}$ & $n^{6}$ \\
\hline
\end{tabular}

It is instructive to compare this with fuzzy odd spheres

\begin{tabular}{|c|c|c|c|c|}
\hline $\begin{array}{c}\text { Fuzzy sphere } \\
S^{D} \\
D=2 k-1\end{array}$ & $\begin{array}{c}n \text {-scaling of } \\
\text { Radius } \\
R \sim n\end{array}$ & $\begin{array}{c}n \text {-scaling of } \\
\text { geometrical } \\
\text { dof } n^{D}\end{array}$ & $\begin{array}{c}n \text {-scaling of } \\
\text { total dof } N^{2} \sim n^{T} \\
=n^{k^{2}+k-2}\end{array}$ & $\begin{array}{c}\text { n-scaling of } \\
\text { excess dof } \\
n^{T-D}\end{array}$ \\
\hline$S^{3}$ & $n$ & $n^{3}$ & $n^{4}$ & $n$ \\
\hline$S^{5}$ & $n$ & $n^{5}$ & $n^{10}$ & $n^{5}$ \\
\hline$S^{7}$ & $n$ & $n^{7}$ & $n^{18}$ & $n^{11}$ \\
\hline$S^{9}$ & $n$ & $n^{9}$ & $n^{28}$ & $n^{19}$ \\
\hline
\end{tabular}

The first five columns for even and odd spheres are the same, and the odd sphere analog of the last column is conspicuously absent, since we have not been able to decipher the nature of the gauge theory living on the odd sphere, although we did give an outline of a field theory on the higher dimensional coset in section 7 . Since the number of extra degrees of freedom scales like an odd power of $n$, assuming one could make sense of the theory on the odd sphere as some sort of generalized non-abelian gauge theory, the rank would be $n^{p / 2}$ for $p$ odd. Curiously, a completely different line of argument, also motivated by an attempt to understand 5-branes, leads one to consider a generalization of non-abelian 
gauge theory where the number of degrees of freedom scales like $k^{3}$ rather than $k^{2}$ where $k$ is an integer number of branes. This famous exponent appears in trying to account for the entropy of five-branes [35]. The exponent $n^{5}$ should be compared to the odd power of $k$ 1. It would be interesting to find a scenario where the appearance of odd powers of a brane number as the counting of degrees of freedom of a field theory on a fuzzy odd sphere can be related to the entropy of five branes.

\subsection{Further comments and open questions}

The results of [2] 11] [4] [12] show that a lot of interesting physics of spherical 4-branes constructed from 0 branes can be inferred directly from the Matrices $X_{i}$ satisfying the defining equation of an even sphere. It is also clear that the higher dimensional fuzzy spheres give a neat generalization of the physics of fuzzy 4-spheres [12] [16]. In this paper, we hope to have convinced the reader that by considering a natural generalization of the fuzzy even sphere construction to the odd sphere case as in 10 we can find many features similar to the fuzzy even sphere case, e.g the relevance of field theories on higher dimensional cosets. Admittedly these field theories are somewhat more complicated and even the sketch of a reduction to a theory on the sphere itself has not been accomplished ( unlike the even sphere case ). However the fact that the higher dimensional cosets have a rather rich geometry related to symmetric spaces, as explained in section 9.1 suggests that these fuzzy odd sphere Matrix constructions should have a role similar in at least some respects to fuzzy even spheres. One question in the direction of finding this physics is to find a SUSY Matrix quantum mechanics model, perhaps allowing the generalized type of SUSY considered in [5], which would admit any of the fuzzy odd spheres as a SUSY preserving solution. It was observed in [5], using simple scaling arguments, that one did not expect a five sphere to appear as a classical solution. It would be interesting to find any M-theory background with a Matrix model dual where the duality would predict the existence, rather the non-existence, of fuzzy five spheres. This may be viewed as a reformulation of the transverse 5-brane problem of BFSS Matrix theory.

1 I thank Savdeep Sethi for discussions along these lines 


\section{Acknowledgements}

I am happy to acknowledge interesting discussions with Bobby Acharya, Steve Corley, Jerome Gauntlett, Zack Guralnik, George Papadopoulos, Chris Hull, Antal Jevicki, Robert de Mello Koch, Rob Myers, Savdeep Sethi, Wati Taylor. This research was supported by DOE grant DE-FG02/19ER40688-(Task A). I would like to thank the Isaac Newton Institute for hospitality while part of this work was done.

\section{Appendix 1 : Notation for Gamma matrices}

We recall the explicit construction of the $\Gamma$ matrices. To fix notation we will give the form of the Gamma matrices $\Gamma_{\mu}$ with $\mu=1 \cdots 2 k+1$. The Gamma Matrices of $S O(2 k+1)$ obey the equations :

$$
\left[\Gamma_{\mu}, \Gamma_{\nu}\right]=\delta_{\mu \nu}
$$

They can be expressed in terms of a set of fermionic oscillators $a_{i}$ with $i$ running from 1 to $k$, and obeying

$$
\left\{a_{i}, a_{j}^{\dagger}\right\}=\delta_{i j}
$$

- The expressions for the Gamma Matrices are :

$$
\begin{aligned}
& \Gamma_{2 i-1}=\left(a_{i}+a_{i}^{\dagger}\right) \\
& \Gamma_{2 i}=i\left(a_{i}-a_{i}^{\dagger}\right)
\end{aligned}
$$

for $i=1 \cdots k$ and $\Gamma_{2 k+1}=i^{k} \Gamma^{1} \cdots \Gamma^{2 k}$. From (9.5) it follows that $\Gamma_{2 i-1} \Gamma_{2 i}=i\left[a_{i}^{\dagger}, a_{i}\right]$.

A $2^{k}$ dimensional representation of the $\Gamma$ matrix algebra is obtained by defining a state $v_{0}$ which is annihilated by the fermionic annihilation operators $a_{i}$ and acting with the creation operators to generate $2^{k}$ different states. Using the abobe we find $\Gamma^{5} v_{0}=v_{0}$. States in $V_{+}$, the positive chirality representation of $S O(2 k)$, have an even number of $a^{\dagger}$ acting on $v_{0}$. States in $V_{-}$, the negative chirality representation of $S O(2 k)$, have an odd number of $a^{\dagger}$ acting on $v_{0}$. 


\section{References}

[1] D. Kabat and W. Taylor, "Spherical membranes in Matrix theory," hep-th/9711078, Adv.Theor.Math.Phys. 2 (1998) 181-206

[2] J.Castelino, S. Lee and W. Taylor IV, "Longitudinal Five-Branes as Four Spheres in Matrix Theory," Nucl.Phys.B526 (1998) 334, hep-th/9712105.

[3] R. Myers, "Dielectric-Branes," hep-th/9910053

[4] N. Constable, R. Myers, O. Tafjord, "Non-abelian Brane intersections, " hepth/0102080, JHEP 0106 (2001) 023

[5] D. Berenstein, J. Maldacena, H. Nastase "Strings in flat space and pp waves from $\mathcal{N}=4$ Super Yang Mills," hep-th/0202021, JHEP 0204 (2002) 013

[6] J. Maldacena, A. Strominger, "AdS3 Black Holes and a Stringy Exclusion Principle," hep-th/980408, JHEP 9812 (1998) 005

[7] A. Jevicki, S. Ramgoolam, "Non commutative gravity from the ADS/CFT correspondence," hep-th/9902059, JHEP 9904 (1999) 032

[8] P.M. Ho, M. Li, "Fuzzy Spheres in AdS/CFT Correspondence and Holography from Noncommutativity," hep-th/0004072, Nucl.Phys.B596:259-272,2001

[9] M. Berkooz, H. Verlinde "Matrix Theory, AdS/CFT and Higgs-Coulomb Equivalence," hep-th/9907100, JHEP 9911 (1999) 037

[10] Z. Guralnik, S. Ramgoolam "On the Polarization of Unstable D0-Branes into NonCommutative Odd Spheres," hep-th/0101001, JHEP 0102 (2001) 032

[11] S. Ramgoolam, "On spherical harmonics for fuzzy spheres in diverse dimensions, "hep-th/0105006, Nucl.Phys. B610 (2001) 461-488

[12] P.M. Ho, S. Ramgoolam, "Higher dimensional geometries from matrix brane constructions," hepth/0111278, Nucl.Phys. B627 (2002) 266-288

[13] Yi-Xin Chen, Bo-Yu Hou, Bo-Yuan Hou "Non-commutative geometry of 4-dimensional quantum Hall droplet," hep-th/0203095

[14] Y. Kimura "Noncommutative Gauge Theory on Fuzzy Four-Sphere and Matrix Model," hep-th/0204256

[15] S.C Zhang, J. Hu "A Four Dimensional Generalization of the Quantum Hall Effect," cond-mat/0110572, Science 294 (2001) 823

[16] M. Fabinger, "Higher-Dimensional Quantum Hall Effect in String Theory," hepth/0201016, JHEP 0205 (2002) 037

[17] A.P. Balachandran "Quantum Spacetimes in the Year 1," hepth/0203259

[18] A. Salam, J. Strathdee, "On Kaluza-Klein Theory," Ann. Phys. 141, 1982, 216

[19] N.L. Wallach, "Harmonic analysis on homogeneous spaces," M. Drekker Inc. NY 1973

[20] Y. Kazama, H. Suzuki, "New $N=2$ superconformal field theories and superstring compactification" Nucl.Phys.B321:232,1989 
[21] M. Kramer, "Some remarks suggesting an interesting theory of harmonic functions on $S U(2 n+1) / S p(n)$ and $S O(2 n+1) / U(n)$," Arch. Math. $33(1979 / 80), 76-79$.

[22] P.M. Ho, "Fuzzy sphere from Matrix model," hepth/0110165, JHEP 0012 (2000) 015

[23] T. Banks, W. Fischler, S. Shenker, L. Susskind, "M-Theory as a Matrix model : A conjecture," hep-th/9610043, Phys.Rev.D55:5112-5128,1997

[24] N. Ishibashi, H. Kawai, Y. Kitazawa, A. Tsuchiya, " A large-N reduced model as Superstring, " Nucl. Phys. B498 (1997) 467-491

[25] V. Periwal, "Matrices on a point as the theory of everything," Phys.Rev. D55 (1997) 1711-1713

[26] S. Chaudhuri, "Bosonic Matrix Theory and D-branes," hepth/0205306

[27] M. Bagnoud, L. Carlevaro, A. Bilal, "Supermatrix models for M-theory based on $\operatorname{osp}(1-32, \mathrm{R}), "$ hep-th/0201183

[28] L. Smolin, "M theory as a matrix extension of Chern Simons theory," hepth/0002009, Nucl.Phys.B591:227-242,2000

[29] I. Bandos, J. Lukierski, "New superparticle models outside the HLS suersymmetry scheme," hep-th/9812074

[30] S.Iso, Y.Kimura, K.Tanaka, K. Wakatsuki, "Noncommutative Gauge Theory on Fuzzy Sphere from Matrix Model," hep-th/0101102

[31] W. Fulton and G. Harris, "Representation theory," Springer Verlag 1991.

[32] M. Atiyah and E. Witten, "M-Theory Dynamics On A Manifold Of $G_{2}$ Holonomy," hep-th/0107177

[33] S. Ramgoolam, D. Waldram, "Zero branes on a compact orbifold," hep-th/9805191, JHEP 9807:009,1998

[34] Brian R. Greene, C.I. Lazaroiu, Piljin Yi " D Particles on $T^{4} / Z(N)$ Orbifolds and their resolutions," hep-th/9807040, Nucl.Phys.B539:135-165,1999

[35] I. Klebanov, A. Tseytlin, "Entropy of Near-Extremal Black p-branes," hep-th/9604089, Nucl.Phys. B475 (1996) 164-178 